\title{
Antioxidant or neurotrophic factor treatment preserves function in a mouse model of neovascularization-associated oxidative stress
}

\author{
Michael I. Dorrell, ${ }^{1}$ Edith Aguilar, ${ }^{1}$ Ruth Jacobson, ${ }^{1}$ Oscar Yanes, ${ }^{2}$ Ray Gariano, ${ }^{1}$ \\ John Heckenlively, ${ }^{3}$ Eyal Banin, ${ }^{4}$ G. Anthony Ramirez, ${ }^{5}$ Mehdi Gasmi, ${ }^{5}$ Alan Bird, ${ }^{6}$ \\ Gary Siuzdak, ${ }^{2}$ and Martin Friedlander ${ }^{1}$
}

\begin{abstract}
${ }^{1}$ Department of Cell Biology and 2Department of Molecular Biology, Scripps Center for Mass Spectrometry, The Scripps Research Institute, La Jolla, California, USA. ${ }^{3}$ Department of Ophthalmology, Kellogg Eye Institute, University of Michigan, Ann Arbor, Michigan, USA. ${ }^{4}$ Department of Ophthalmology, Hadassah-Hebrew University Medical Center, Jerusalem, Israel. ${ }^{5}$ Ceregene, San Diego, California, USA. 6 Institute of Ophthalmology, Moorfields Eye Hospital, University College London, London, United Kingdom.
\end{abstract}

\begin{abstract}
In several disease states, abnormal growth of blood vessels is associated with local neuronal degeneration. This is particularly true in ocular diseases such as retinal angiomatous proliferation (RAP) and macular telangiectasia (MacTel), in which, despite the absence of large-scale leakage or hemorrhage, abnormal neovascularization (NV) is associated with local neuronal dysfunction. We describe here a retinal phenotype in mice with dysfunctional receptors for VLDL (Vldlr ${ }^{-/-}$mice) that closely resembles human retinal diseases in which abnormal intra- and subretinal NV is associated with photoreceptor cell death. Such cell death was evidenced by decreased cone and, to a lesser extent, rod opsin expression and abnormal electroretinograms. Cell death in the region of intraretinal vascular abnormalities was associated with an increased presence of markers associated with oxidative stress. Oral antioxidant supplementation protected against photoreceptor degeneration and preserved retinal function, despite the continued presence of abnormal intra-and subretinal vessels. What we believe to be novel, Müller cell-based, virally mediated delivery of neurotrophic compounds specifically to sites of NV was also neuroprotective. These observations demonstrate that neuronal loss secondary to NV can be prevented by the use of simple antioxidant dietary measures or cell-based delivery of neurotrophic factors, even when the underlying vascular phenotype is not altered.
\end{abstract}

\section{Introduction}

Oxidative damage is associated with the pathogenesis of multiple degenerative disorders of the CNS, including Parkinson disease (1), Huntington disease (2), and Alzheimer disease $(3,4)$. The pathogenesis of these and other degenerative diseases is associated with oxidative stress due to multiple factors, including excessive neovascularization (NV) (5). Although the direct relationship between NV and neurodegeneration remains unclear, the presence of excess vessels may exacerbate the underlying neurodegeneration and contribute to progression of the disease (1). A growing body of evidence suggests that abnormalities in small blood vessels can have a deleterious effect on neighboring neurons. This effect may be the result of disruption of vascular/parenchymal trophic interactions (6), deleterious activity of inflammatory cells associated with NV (7), or accumulation of reactive oxygen species. Cone photoreceptors can be especially sensitive to oxidative damage, at least in part because of the stressful and naturally oxidative environment of the retina (8-10). In the presence of abnormal NV, normal

Conflict of interest: G. Anthony Ramirez and Mehdi Gasmi are employees of Ceregene. Nonstandard abbreviations used: AAV-2, serotype 2 adeno-associated virus; AMD, age-related macular degeneration; ERG, electroretinography, electroretinographies; GFAP, glial fibrillary acidic protein; INL, inner nuclear layer; MacTel, macular telangiectasia; NT4, neurotrophin-4; NV, neovascularization; ONL, outer nuclear layer; OP, oscillatory potential; RAP, retinal angiomatous proliferation; RPE, retinal pigment epithelium; VLDLR, VLDL receptor.

Citation for this article: J. Clin. Invest. 119:611-623 (2009). doi:10.1172/JCI35977. oxidative stress defense mechanisms may no longer be adequate, leading to cell death.

The growth of blood vessels in the mammalian eye is highly regulated, but abnormal retinal angiogenesis is frequently associated with the most common causes of vision loss in industrialized nations (11). In a relatively uncommon, but probably underdiagnosed, form of retinal NV called macular telangiectasia (MacTel; also referred to as idiopathic parafoveal telangiectasia), intraretinal telangiectatic vessels proliferate in the central portion of the inner retina and grow into the normally avascular outer retina (12-14). In the NV form of age-related macular degeneration (AMD), abnormal vessels typically arise from the choroid and invade the subretinal space (15). However, in a subset of patients with AMD, intraretinal and subretinal NV arises from the inner retinal vessels, a condition known as retinal angiomatous proliferation (RAP) (16). Both MacTel and RAP exhibit glial cell abnormalities and varying degrees of photoreceptor degeneration associated with abnormal intra- and subretinal NV $(12,14)$.

Subretinal NV originating from the intraretinal vessels can be generated by blocking R-cadherin-mediated endothelial cell migratory cues $(17,18)$ or by overexpressing VEGF in photoreceptors (19). Similarly misdirected vascular growths occur in the retinas of ldllr $^{-1-}$ mice, which have a defective gene for the VLDL receptor (VLDLR; refs. 20-22). Aberrant retinal vessels in these mice extend from the inner retina through the photoreceptors to the subretinal space. Here we describe the retinal phenotype in eyes 

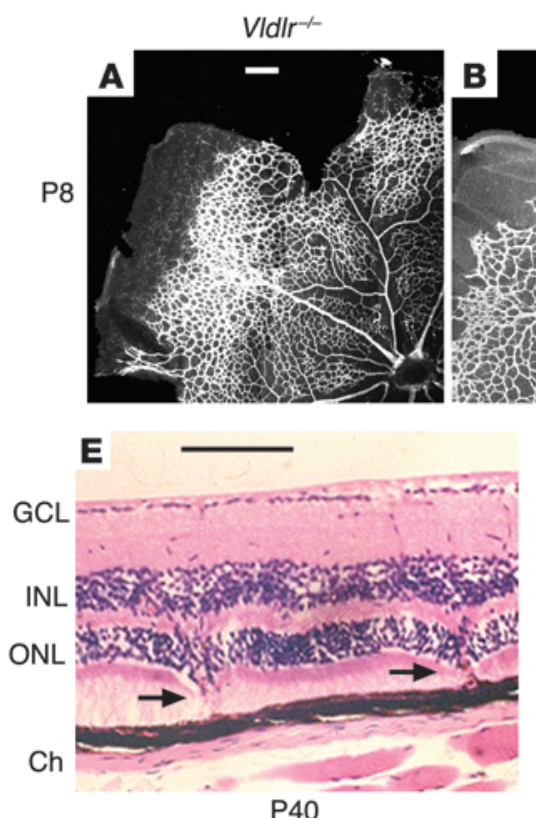

WT
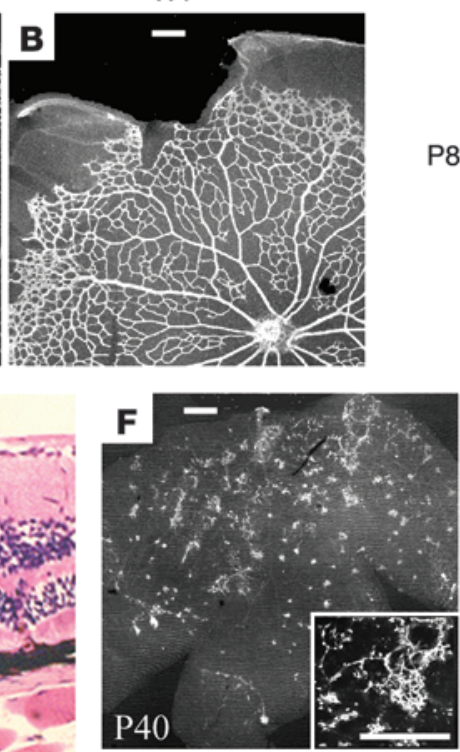
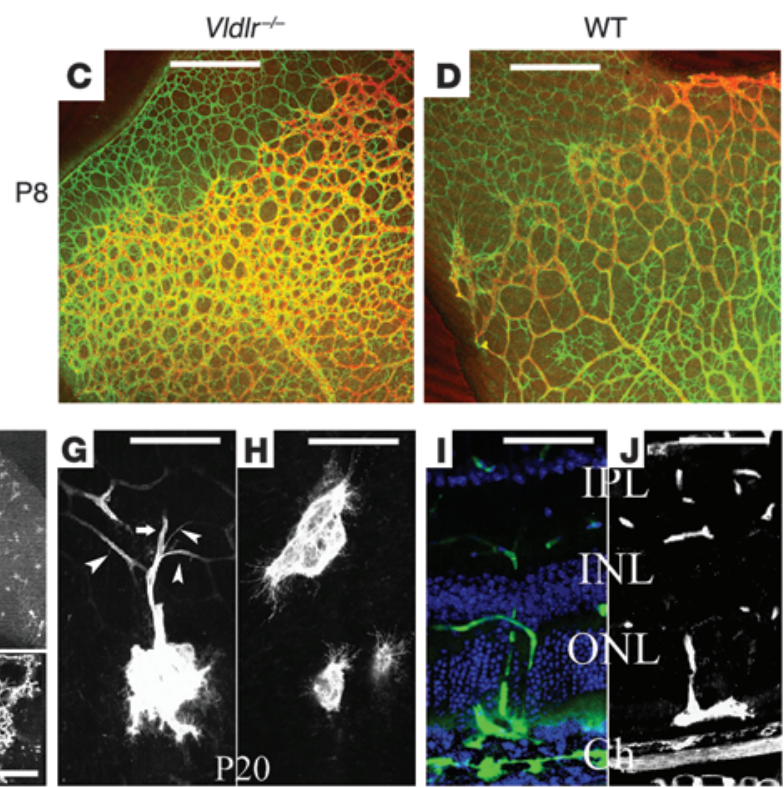

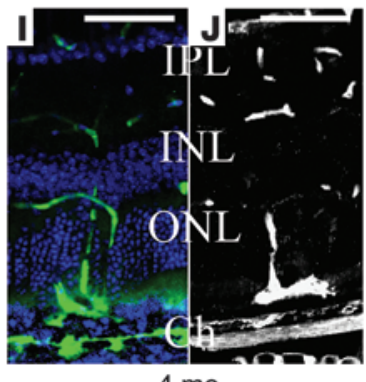

$4 \mathrm{mo}$

Figure 1

Intraretinal vascular abnormalities in VIdlr $r^{-1}$ mouse retinas. (A and B) The developing superficial vascular plexus of VIdlr ${ }^{-1-}$ retinas was hyperdense, particularly in the periphery, at P8. (C and D) Peripheral hypervascularity (isolectin GS; red) was associated with increased density of astrocytes (GFAP; green). (E) H\&E-stained retinal sections from a P40 VIdlr-l- mouse showed intraretinal vessels (arrows) that originated from the inner retinal vascular plexuses and migrated through the photoreceptors to the subretinal space. GCL, ganglion cell layer; Ch, choroid. (F) These intraretinal vessels formed retinal-retinal anastomoses throughout the central two-thirds of the VIdlr-/- retinas. Inset shows a higher-magnification view. (G) Intraretinal vessels originated from multiple sprouts off the deep (arrowheads) and intermediate vascular plexuses (arrow) and could form large angiomatous structures within the subretinal space. (H) Filopodia extended from subretinal vascular endothelial cells and extended laterally to form retinal vascular anastomoses. (I and $\mathbf{J}$ ) Fluorescein angiography (I) or isolectin GS staining ( $\mathbf{J})$ demonstrated the retinal origin of the intraretinal and subretinal NV. IPL, inner plexiform layer. Scale bars: $100 \mu \mathrm{m}$.

from $V l d l r^{-/-}$mice and its striking similarity to that observed in human patients with RAP or MacTel, including focal disruption of photoreceptors coincident with the abnormal penetrating vessels. Using the Vldlr ${ }^{-1-}$ mouse model, we also demonstrated a correlation between intraretinal vascular abnormalities, oxidative stress, and cone-dominated neuronal degeneration, an association that may also be observed in other neuronal degenerative disorders. Targeted delivery of neurotrophic factors to sites of abnormal NV or oral antioxidant supplementation significantly reduced photoreceptor degeneration and protected against visual dysfunction in $\mathrm{Vldl}^{-/-}$ mice. We hypothesize that diet-responsive, NV-induced oxidative stress may similarly link retinal angiogenesis and neuronal loss in human diseases. In addition, we demonstrated that intravitreally injected glial fibrillary acidic protein (GFAP) promoter-driven serotype 2 adeno-associated viruses (AAV-2s) targeted to activated Müller glia delivered neurotrophic factors to photoreceptors located in the outer retina via the retinal spanning Müller cell processes. Our findings reveal what we believe to be a novel treatment modality by which ocular gene therapy may be used to treat similar ocular vascular diseases with associated neuronal degeneration.

\section{Results}

Characterization of abnormal retinal NV in Vldlr-/- mice. Vldlr-/- mice appear outwardly normal, and are viable and fertile (23), but exhibit abnormalities in the retinal vasculature $(20,21)$. Systematic evaluation of neonatal $\mathrm{Vldlr}^{-/-}$mice revealed normal development of retinal vessels through the first postnatal week. During the second postna- tal week, $\mathrm{Vldlr}^{-/}$- retinas exhibited transient hyperproliferation of the inner vascular plexus and associated astrocytes, particularly in newly vascularized regions near the retinal periphery (Figure 1, A-D). This associated astrocytosis is consistent with previous studies demonstrating a direct correlation between vessels and astrocytes in the inner retina $(17,24,25)$. By the third postnatal week, vessel and astrocyte density in the superficial plexus receded to normal levels.

Dramatic abnormalities were observed during formation of the deep vascular plexuses in $\mathrm{Vldl}^{-/-}$mice. During the second postnatal week, abnormal intraretinal vessels sprouted from the deep and intermediate plexuses and penetrated through the outer nuclear layer (ONL) to the subretinal space (Figure 1, E-J). By 4 weeks of age, numerous intraretinal vessels extended to form a subretinal vascular network (Figure 1F). Choroidal vessels were normal at this time, and breaks in Bruch's membrane and anastomoses of the intraretinal vessels with the choriocapillaris were not observed in mice less than 6 months of age, confirming the intraretinal origin of subretinal NV (Figure 1, I and J). Although the numbers of subretinal sprouts were reduced in older mice, most intra- and subretinal vessels persisted throughout the life of the $\mathrm{Vldlr}^{-/}$mice.

The subretinal vessels were patent and perfused (Figure 1I and Figure 2, A and B). Modest extravasation of 43-kDa fluorescein dextran was observed from a subset of subretinal vascular sprouts (Figure 2, A-C), indicating a mild dysfunction of the blood-retinal barrier normally formed by tight junctions between nonfenestrated vascular endothelial cells and close association with perivascular glia (26). Electron microscopic analysis of intra- and subretinal NV 

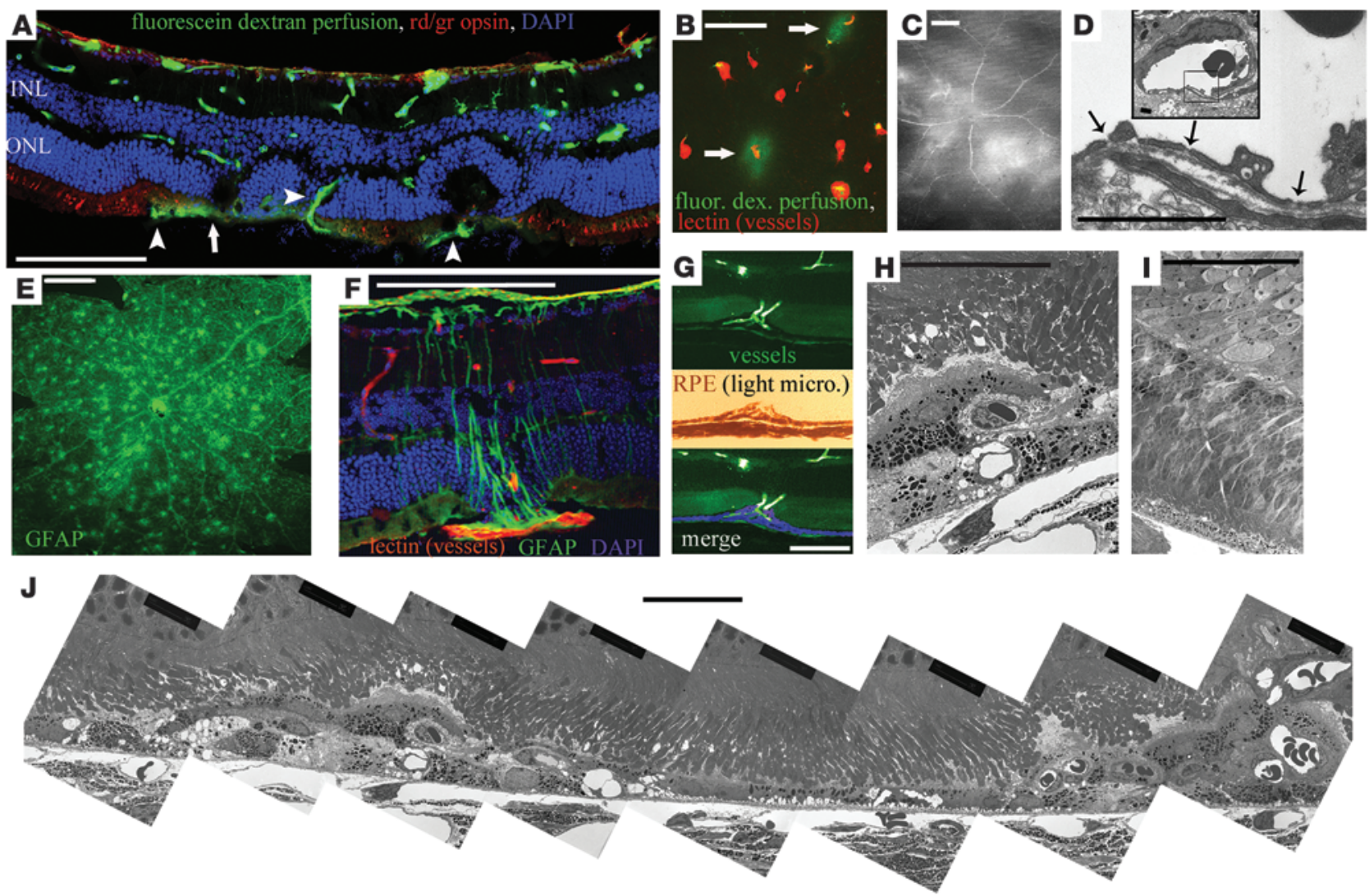

Figure 2

Characterization of abnormal NV in the VIdlr ${ }^{-1-}$ mouse retina. (A) Retinal cross-section montage demonstrating multiple retinal abnormalities in the VIdlr $r^{-1}$ mouse retina at 3 months of age, including subretinal vessels (arrowheads), vascular leak (arrow), abnormal retinal morphology, abnormal neuronal layering in the ONL and INL, and loss of red/green ( $\mathrm{rd} / \mathrm{gr}$ ) cone opsin staining. (B and C) Vascular leak was associated with a small subset of subretinal vessels, as demonstrated by extravasation of perfused 43-kDa fluorescein-labeled dextran. (D) Fenestrae within endothelial cells of the subretinal vessels were observed by electron microscopy. Inset shows a lower-magnification view, in which the boxed region defines the bounds of the higher-magnification view. (E) Punctate GFAP staining in retinal whole mounts demonstrated glial activation associated with abnormal NV in the VIdlr-/- mouse retinas. (F) This punctate staining represented Müller cells specifically activated around the abnormal intraretinal vessels. ( $G$ and $\mathbf{H}$ ) The RPE formed multicellular layers that engulfed abnormal intraretinal vessels. (I) Electron microscopy demonstrated normal retinal morphology at P12, just prior to NV onset. (J) Montage of multiple electron microscopy images showing retinal damage spatially associated with abnormal NV in 2 separate regions, while intermittent retinal morphology between lesions remained mostly normal. Scale bars: $100 \mu \mathrm{m}(\mathbf{A}-\mathbf{C}$ and $\mathbf{E}-\mathbf{G}) ; 50 \mu \mathrm{m}(\mathbf{H}-\mathbf{J}) ; 10 \mu \mathrm{m}$ (D).

in $\mathrm{Vldlr}^{-/}$mouse retinas revealed fenestrated endothelia (Figure 2D), providing an anatomic basis for the abnormal permeability observed in these vessels. Müller glial cell activation, as demonstrated by GFAP expression, was selectively associated with, but secondary to, the formation of intraretinal NV (Figure 2, E and F).

By the fifth postnatal week, the normal retinal pigment epithelium (RPE) monolayer was disrupted in areas surrounding subretinal vascular lesions, and multiple layers of RPE cells enveloped the neovascular complexes in $\mathrm{Vldlr}^{-/-}$retinas (Figure 2, G and H). These abnormalities lead to focal distortion and scarring of the retina, and were found to be associated with focal loss of photoreceptor inner and outer segments, as demonstrated by abnormal retinal morphology and an absence of red/green opsin staining in regions with subretinal NV (Figure 2, A and J). Neuronal abnormalities never preceded the appearance of intra- and subretinal NV. At $\mathrm{P} 12$, just prior to the onset of abnormal NV, all retinal layers were intact and identical to those of age-matched WT C57BL/6J controls when evaluated by electron and confocal microscopy (Figure
2I). Thus, neuronal abnormalities in $\mathrm{Vldlr}^{-/-}$mouse retinas appear to be the result, rather than a cause, of NV.

Subretinal NV in Vldlr-/- retinas results from increased VEGF. In order to gain insight into a possible relationship between VLDLR deficiency and the development of abnormal intraretinal vessels, we analyzed VLDLR expression in WT mouse retinas. The ganglion cell layer, retinal vessels, inner nuclear layer (INL), ONL, photoreceptor inner and outer segments, and RPE were isolated using laser capture microdissection techniques. Each of these fractions is highly enriched for the indicated retinal layer, although on occasion, neighboring cells may have been inadvertently captured as a result of technical limitations with the technique. RNA was purified and analyzed by quantitative RT-PCR to assess the relative levels of VLDLR expression in each retinal layer. At P14, when intraretinal NV began in Vldlr ${ }^{-/-}$retinas, VLDLR expression in WT mice was greatest in the photoreceptors, with the RPE also expressing high levels of VLDLR mRNA. Expression in other retinal tissues, including retinal vessels, was observed at relatively low levels (Figure 3A). 

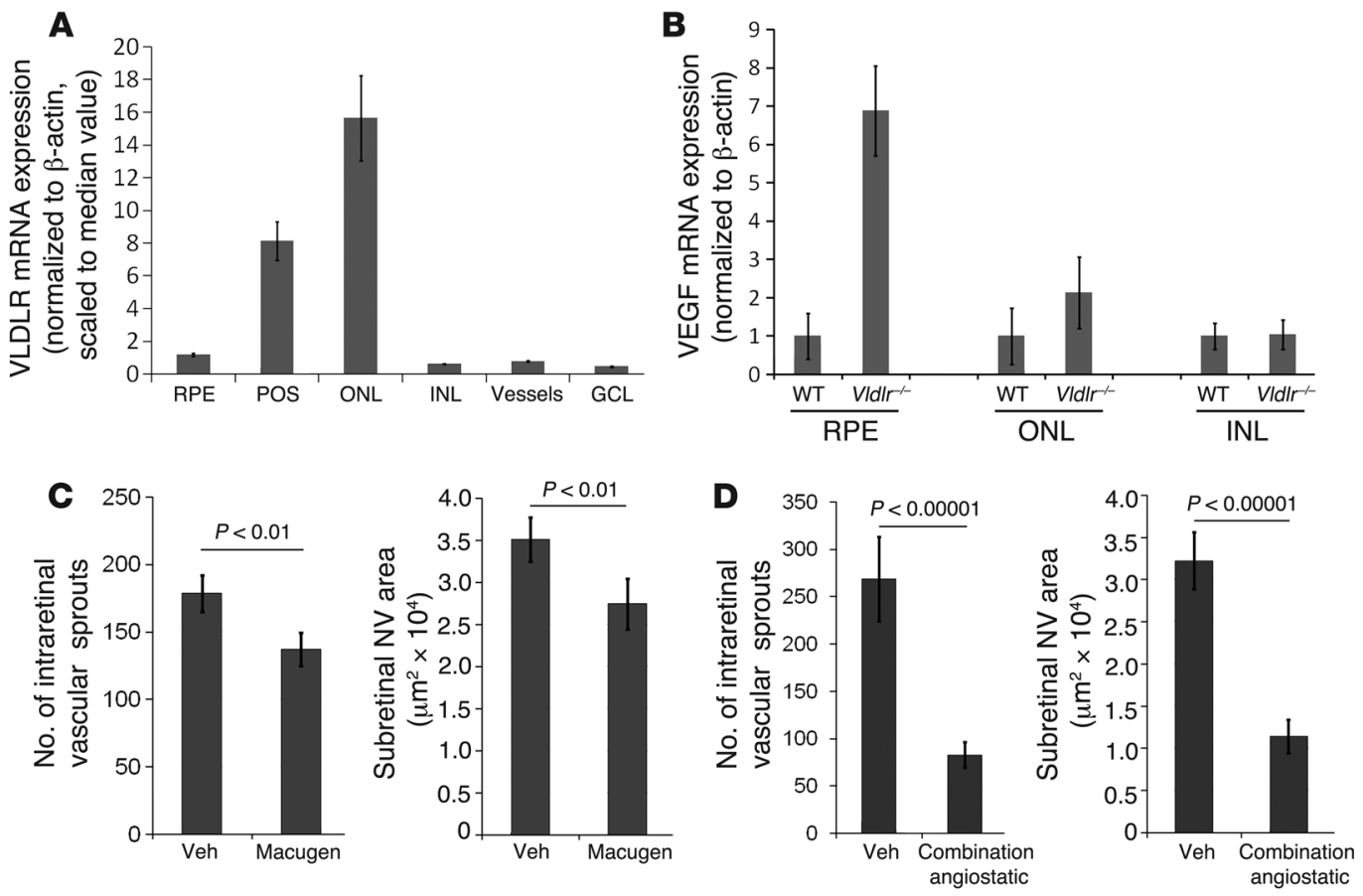

E
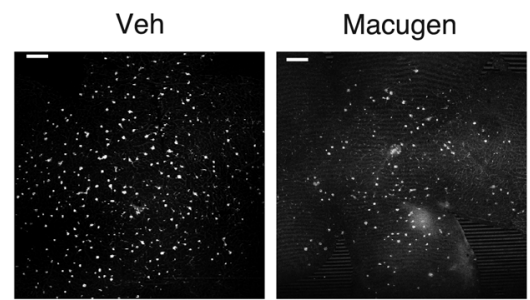

Combination angiostatic

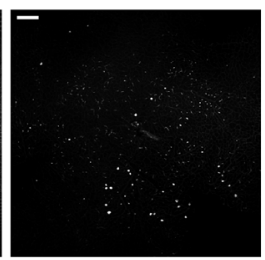

\section{Figure 3}

VEGF upregulation is associated with intraretinal NV in VIdlr-/- mice. (A) Most VLDLR expression in laser-captured retinal tissue from P14 C57BL6/J mice occurred in the ONL and photoreceptor segments (POS), with lower expression in the RPE and other retinal tissues (triplicate data). (B) In VIdlr ${ }^{-1}$ retinas, significant upregulation of VEGF occurred within the RPE $(P<0.0001)$ and photoreceptors $(P<0.05)$ at P14 (triplicate data). (C) Blocking VEGF 165 activity with intravitreal Macugen injection at P12 significantly reduced the number of intraretinal NV sprouts and the area of subretinal NV at P20 in VIdlr $r^{-1}$ retinas. $n=10-12$ retinas per group. Veh, vehicle. (D) Combination angiostatic therapy reduced abnormal retinal NV to an even greater extent. $n=10-12$ retinas per group. (E) Whole retina images of vessels, focused within the subretinal space, showed a sharp reduction in intraretinal NV after treatment with Macugen and combination angiostatics. Scale bars: $250 \mu \mathrm{m}$. Error bars denote SEM.

Recently it has been reported that VEGF expression is increased in Vldl $r^{-1-}$ mouse retinas (27). We confirmed this finding and tested for a direct correlation between VLDLR and VEGF expression in individual retinal layers at P14, when subretinal NV begins in Vldlr-/mice. In WT mice, VEGF expression was highest in the ONL, and smaller amounts of VEGF mRNA were found in the RPE and INL (Figure 3B). In $\mathrm{Vldlr}^{-/}$retinas, VEGF expression was upregulated compared with WT retinas more than 5-fold in the RPE and at least 2 -fold in the ONL. No difference in expression levels was found in other retinal layers (Figure 3B).

It is likely that increased VEGF expression is responsible for new vessel growth. We tested this by using anti-VEGF agents to suppress subretinal NV in $\mathrm{Vldlr}^{-/}$- retinas. Intravitreal injection of Macugen, an RNA aptamer that blocks $V_{E G F}$ $_{165}$, reduced intra- and subretinal NV confirming that VEGF upregulation in the deep retina is critical for abnormal vessel formation in the $\mathrm{Vldlr}^{-/-}$mouse (Figure $3 \mathrm{C})$. In order to further evaluate effects of antiangiogenic therapy on subretinal vessels, we used a combination of angiostatic agents previously shown to synergistically inhibit retinal angiogenesis (28): Macugen, an integrin $\alpha_{v} \beta_{3} / \alpha_{v} \beta_{5}$ antagonist, and T2-TrpRS, a fragment of tryptophan transfer RNA synthetase with angiostatic activity (29-31). Using combination therapy, we observed substantial reductions in intraretinal vascular sprouts and total area of subretinal NV (70\% and 60\%, respectively; Figure 3, D and E).

Photoreceptor degeneration in Vldlr ${ }^{-1}$ - mouse eyes. Our initial morphological studies suggested that neuronal abnormalities are directly associated with NV in the Vldlr ${ }^{-1}$ mouse retinas (Figure 2A). These abnormalities consisted of rosettes within the ONL, thinning of the ONL and, to a lesser extent, the INL, and reduced red/green opsin in the areas directly associated with subretinal NV (Figure 4A). To assess photoreceptor degeneration in $\mathrm{Vldlr}^{-/-}$mice further, real-time RT-PCR was performed to analyze opsin-1 (cone-specific) and rhodopsin (rod-specific) mRNA expression in whole retinas. Although an indirect correlation, we believe this measure to be correlated with photoreceptor viability and health, and it allowed analysis of degeneration in whole retinas. In 2-month-old mice, a $40 \%$ decrease 
A 7 mo WT

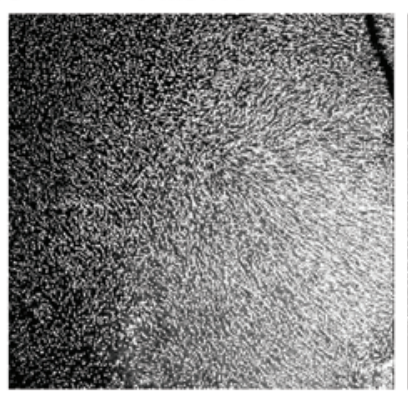

Opsin
$7 \mathrm{mo} \mathrm{Vldlr^{-- }}$
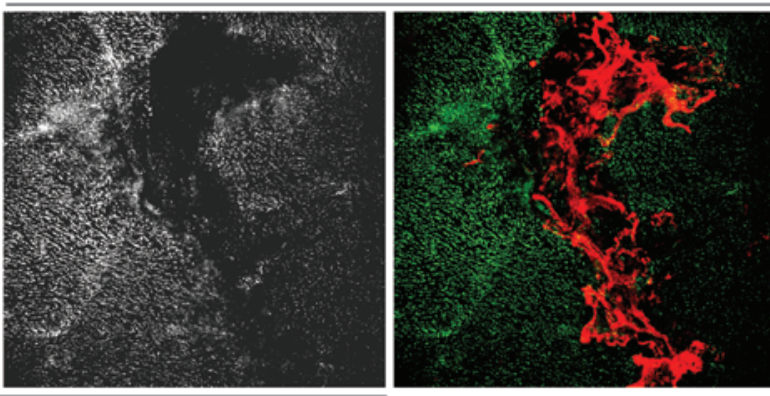

Opsin, vessels
B

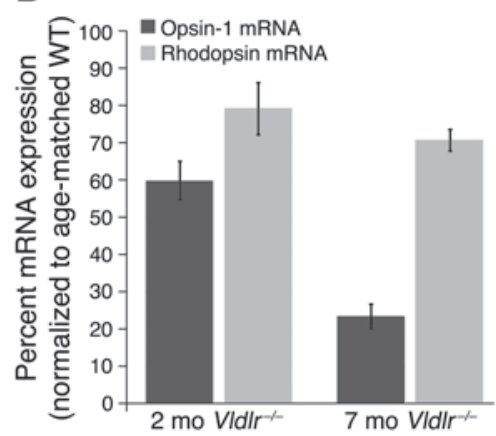

C

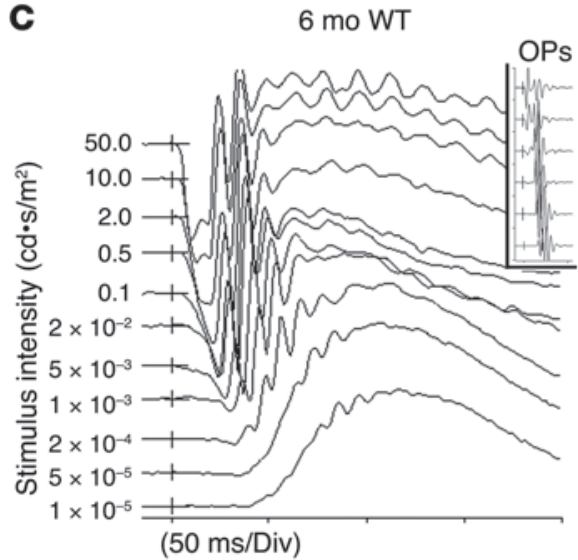

D

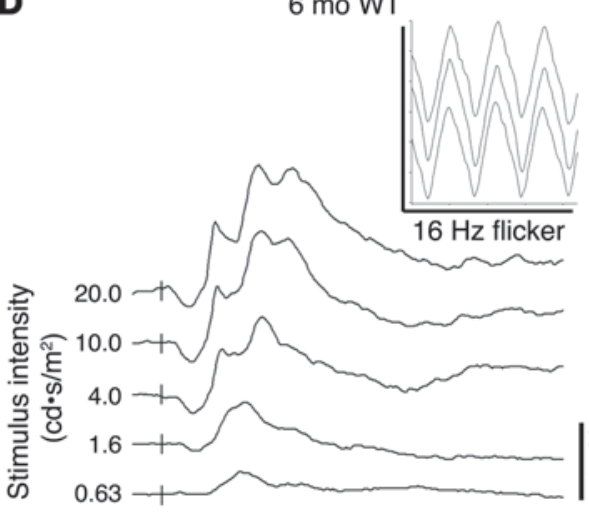

(50 ms/Div)

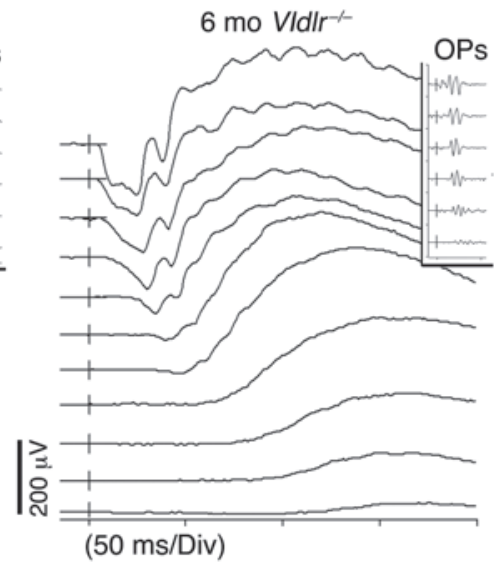

6 mo VIdlr ${ }^{-1-}$

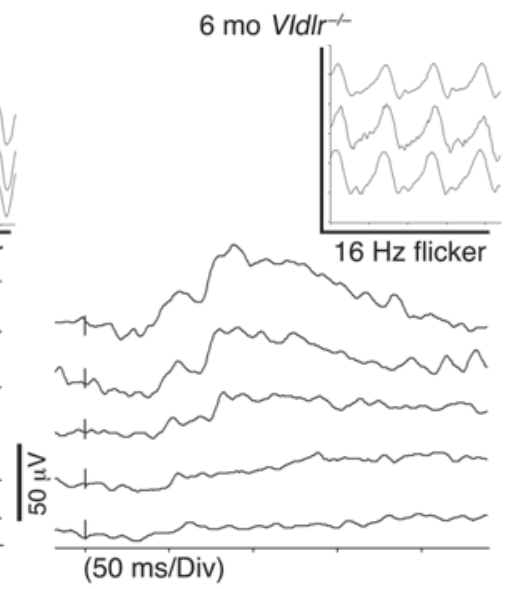

\section{Figure 4}

Photoreceptor degeneration, loss of retinal function, and increased oxidative stress are associated with intraretinal NV in VIdlr ${ }^{-/}$ mouse retinas. (A) Opsin staining was reduced in VIdlr-/- retinal whole mounts, with loss of opsin directly associated with the presence of subretinal NV. Original magnification, $\times 100$. (B) Reduced mRNA production of opsin-1 (cone-specific opsin) and rhodopsin (rod-specific opsin) in retinas of 2- and 7-moold Vldlr-1- mice compared with age-matched C57BL6/J WT controls, indicating a substantial loss of cones and, to a lesser extent, rods. Error bars denote SEM. $n=18$ [2 mo WT], 28 [2 mo VIdll ${ }^{-/}$], 36 [7 mo WT], 30 [7 mo Vldlr--_]. (C) Photoreceptor degeneration resulted in abnormal ERGs, characterized by delayed responses in scotopic measurements and dramatic reductions in the OPs (insets). (D) Photopic ERGs of VIdlr-/mice were also characterized by reduced and delayed responses, with particularly reduced responses to light flicker (insets), a specific measurement of cone activity. in opsin-1 expression was observed in the Vldlr $^{-/}$retinas compared with retinas of age-matched WT controls (Figure 4B). Rhodopsin expression was decreased in $\mathrm{Vldl}^{-1-}$ mouse retinas by only $20 \%$. In 7-month-old mice, cone opsin-1 expression decreased approximately $70 \%$ in $\mathrm{Vldlr}^{-/-}$retinas compared with those of age-matched WT controls, while rhodopsin expression was reduced by $30 \%$ (Figure 4B). Thus, both rod and cone photoreceptor abnormalities were associated with the vascular phenotype in $\mathrm{Vldlr}^{-1-}$ mice, with cones affected earlier and to a greater extent than rods.

Electroretinography (ERG) was performed to compare visual function in $\mathrm{Vldl}^{-1-}$ mice and age-matched WT controls. The ERGs of 6-month-old Vldlr ${ }^{-1-}$ mice exhibited delayed signaling, reduced oscillatory potentials (OPs), and reduced and delayed cone responses. Amplitudes of scotopic rod-dominated responses were largely preserved. However, maximal dark-adapted B-wave implicit times in $\mathrm{Vldlr}^{-/}$mice were consistently prolonged (Figure 4C and Table 1), implying generalized rod dysfunction. Photopic cone responses were also delayed in $V_{l d l r^{-/}}$mice, but unlike the scotopic signals, amplitudes were significantly reduced as well (Figure $4 \mathrm{D}$ and Table 1). Abnormalities in the cone-specific responses to light flicker at $16 \mathrm{~Hz}$ were even more pronounced in the $\mathrm{Vldlr}^{-/}$ mice, with delayed implicit times as well as reduced signal amplitudes (Figure 4D, insets, and Table 1). OPs were also significantly reduced in all 6-month-old Vldlr ${ }^{-/-}$ERGs (Figure 4C, insets, and Table 1), indicating broad retinal signaling defects and suggestive of specific defects in the INL neurons.

Targeted delivery of neurotrophin-4 protects retinas from neuronal degeneration. Although we observed a highly significant reduction in the 
Table 1

Responses to light stimuli in 6-month-old WT and VIdlr-1- mice

\begin{tabular}{|c|c|c|c|c|}
\hline Stimulus & Measurement & $\begin{array}{c}\text { WT } \\
(n=10)\end{array}$ & $\begin{array}{l}\text { VIdIr-/ } \\
(n=12)\end{array}$ & $P$ \\
\hline \multicolumn{5}{|c|}{ ERG scotopic measurements ${ }^{A}$} \\
\hline Low intensityB & B-wave amplitude $(\mu \mathrm{V})$ & $218 \pm 36$ & $252 \pm 28$ & 0.228 \\
\hline Low intensity & B-wave implicit time (ms) & $128 \pm 9$ & $152 \pm 4$ & $0.007^{H}$ \\
\hline High intensityc & A-wave amplitude $(\mu \mathrm{V})$ & $133 \pm 20$ & $78 \pm 8$ & $0.007^{H}$ \\
\hline High intensity & A-wave implicit time (ms) & $27 \pm 2$ & $31 \pm 1$ & $0.042^{\mathrm{H}}$ \\
\hline High intensity & B-wave amplitude $(\mu \mathrm{V})$ & $269 \pm 41$ & $277 \pm 25$ & 0.436 \\
\hline High intensity & B-wave implicit time (ms) & $86 \pm 6$ & $110 \pm 4$ & $0.0008^{H}$ \\
\hline Maximum OPD & Amplitude $(\mu \mathrm{V})$ & $65 \pm 18$ & $21 \pm 3$ & $0.008^{H}$ \\
\hline \multicolumn{5}{|c|}{ Photopic measurements ${ }^{\mathrm{E}}$} \\
\hline $1 \mathrm{~Hz}^{\mathrm{F}}$ & A-wave amplitude $(\mu \mathrm{V})$ & $7.6 \pm 0.8$ & $9.5 \pm 1.2$ & $0.011^{H}$ \\
\hline $1 \mathrm{~Hz}$ & A-wave implicit time (ms) & $24 \pm 2$ & $32 \pm 2$ & $0.008^{H}$ \\
\hline $1 \mathrm{~Hz}$ & B-wave amplitude $(\mu \mathrm{V})$ & $109 \pm 12$ & $87 \pm 6$ & $0.046^{\mathrm{H}}$ \\
\hline $1 \mathrm{~Hz}$ & B-wave implicit time (ms) & $68 \pm 7$ & $89 \pm 4$ & $0.015^{\mathrm{H}}$ \\
\hline $16 \mathrm{~Hz}$ flicker ${ }^{\mathrm{G}}$ & Amplitude $(\mu \mathrm{V})$ & $15.3 \pm 1.6$ & $10.4 \pm 0.8$ & $0.003^{H}$ \\
\hline $16 \mathrm{~Hz}$ flicker & Implicit time (ms) & $64 \pm 4$ & $79 \pm 2$ & $0.0004^{\mathrm{H}}$ \\
\hline
\end{tabular}

Values are mean \pm SEM. ADark-adapted measurements performed to determine rod and mixed cone-rod function. ${ }^{B} 0.005 \mathrm{~cd} \bullet \mathrm{s} / \mathrm{m}^{2}$. ${ }^{\mathrm{C}} 2 \mathrm{~cd} \bullet \mathrm{s} / \mathrm{m}^{2}$. ${ }^{\mathrm{D}} 10 \mathrm{~cd} \bullet \mathrm{s} / \mathrm{m}^{2}$. ELight-adapted measurements performed to determine cone function. $\mathrm{F} 20 \mathrm{~cd} \bullet \mathrm{s} / \mathrm{m}^{2}$. G10 $\mathrm{cd} \bullet \mathrm{s} / \mathrm{m}^{2}$. HStatistically significant difference between WT and VId/r-/mouse retinas.

driven expression of NT4 (AAV-GFAP-NT4) resulted in NT4 production in activated Müller cell bodies and, more importantly, outer retinal processes specifically adjacent to intraretinal NV (Figure 5, E and F). This caused accumulation of NT4 in the outer retina near areas of subretinal NV throughout the central two-thirds of the retina (Figure 5, F and G). Use of AAV-GFAP-NT4 protected the retina from neuronal degeneration, as demonstrated by opsin and rhodopsin mRNA expression (Figure $5 \mathrm{H}$ ), and protected the $V l d l r^{-/}$retinas from the characteristic loss of retinal function, resulting in more normalized ERGs (Figure 5, I and J, and Table 2). This protection, provided by the selective delivery of a neurotrophic factor to retinal areas with subretinal NV, supports a direct correlation between abnormal NV and neuronal degeneration in the retina. Furthermore, because activation of Müller glia is associated with numerous retinal diseases, particularly those associated with abnormal NV and retinal degeneration, our findings provide data supporting the use of activated Müller glia for viral-mediated (GFAP vectors) delivery of therapeutic gene products to the outer retina using intravitreal injections.

Vldlr ${ }^{-1-}$ retinas demonstrate increased oxidative stress. We hypothesized that photoreceptor degeneration might be caused by oxidative stress generated by the presence of abnormal intra- and subretinal vessels. Metabolomic analysis comparing retinas from 4-month-old Vldlr $^{-1-}$ and WT

formation of subretinal NV using combination angiostatic therapy, this effect was only transient, and subretinal NV returned to normal Vldlr ${ }^{-/-}$levels within 2-3 weeks of treatment. This is similar to clinical observations that report temporary beneficial effects of angiostatic therapy. Thus, additional strategies for protecting the retinal neurons in ocular vascular diseases are still required (32). We next evaluated the potential effects of neurotrophic factors specifically delivered to sites of retinal NV and associated retinal degeneration. Because we observed GFAP activation in Müller cells located adjacent to subretinal NV in the $\mathrm{Vldlr}^{-/}$mouse retina (Figure 2, E and F), we obtained an AAV-2 vector containing transgenes driven by a GFAP promoter. Intravitreal injection of the GFP-encoding vector AAV-GFAP-GFP demonstrated that expression was strictly limited to cells of the inner retina in WT mice (Figure 5A); GFP expression in Müller cell processes was observed, but was limited to the ganglion cell layer and the Müller cell bodies. However, in $V_{l d l r^{--}}$mice, GFP expression was observed in a greater number of Müller cells, specifically within the processes of the activated Müller cells adjacent to areas of subretinal NV. Importantly, the GFP signal extended throughout both inner and outer retinas of $\mathrm{Vldlr}^{-/-}$mice in these activated Müller cells (Figure 5, C and D). In contrast, a control vector with a ubiquitously expressed CAG promoter-driven transgene, AAV-CAG-GFP, demonstrated nonspecific expression of GFP in the inner retina mainly localized to ganglion cells, with minimal expression in the outer retina (Figure 5B). Similar expression patterns were observed when the AAV-2 vectors were injected into 5 -month-old adult mice (data not shown). By targeting activated Müller glia using the AAV-2 vector with a GFAP promoter, we were able to specifically deliver transgene products to the outer retina in areas directly adjacent to subretinal NV.

Neurotrophin-4 (NT4) has been shown to protect neurons in several models of neuronal degeneration, including retinal degeneration $(33,34)$. Similar to the GFP vectors, AAV-2 vectors with GFAP- mice demonstrated a significant increase in metabolites commonly associated with oxidative stress (Table 3 and Supplemental Figure 1). L-Carnitine, L-acetylcarnitine, and their derivatives are naturally occurring antioxidants $(35,36)$ that are upregulated in response to increased oxidative stress $(35,37,38)$. The observed increase in these metabolites in the $V l d l r^{-1}$ retinas indicates an endogenous cellular response to oxidative stress. Acrolein is a commonly used marker of lipid peroxidation resulting from oxidative stress $(9,10$, $39,40)$. Acrolein staining was clearly greater in $\mathrm{Vldlr}^{-1-}$ retinas compared with age-matched WT controls (Figure 6, A and B). In the peripheral retina, acrolein levels were similar between $\mathrm{Vldlr}^{-/}$and WT retinas, while acrolein levels were greatly increased in the central two-thirds of Vldlr $^{-1}$ retinas compared with age-matched WT controls (Figure 6C). In $\mathrm{Vldlr}^{-1-}$ eyes, the vast majority of intraretinal NV occurs in the central two-thirds of the retina, whereas the peripheral retinal vasculature remains normal. Thus, increased oxidative stress measured by acrolein staining in $\mathrm{Vldl}^{-/-}$retinas appears to be limited to regions with subretinal NV, supporting a direct relationship between subretinal $\mathrm{NV}$ and oxidative stress.

The transient effect of antiangiogenic treatments in the Vldlr-/retinas rendered us unable to prevent recurrence of subretinal NV using a single angiostatic treatment, and multiple, weekly injections led to phthisis. Thus, it is not possible with this experimental model to determine whether subretinal NV directly causes the observed oxidative stress and associated neuronal degeneration. An alternative hypothesis that might explain the loss of photoreceptors in the presence of increased markers of oxidative stress would be a defect in natural oxidative stress defense mechanisms associated with VLDLR deficiency. To test this hypothesis, we used large-scale genomic analysis to examine the expression of genes encoding factors involved in protection against oxidative stress. Of 210 gene products examined, 109 were found to be expressed in the retina, and 108 of these were equally expressed in 3-week-old $\mathrm{Vldlr}^{-/}$and WT retinas within 

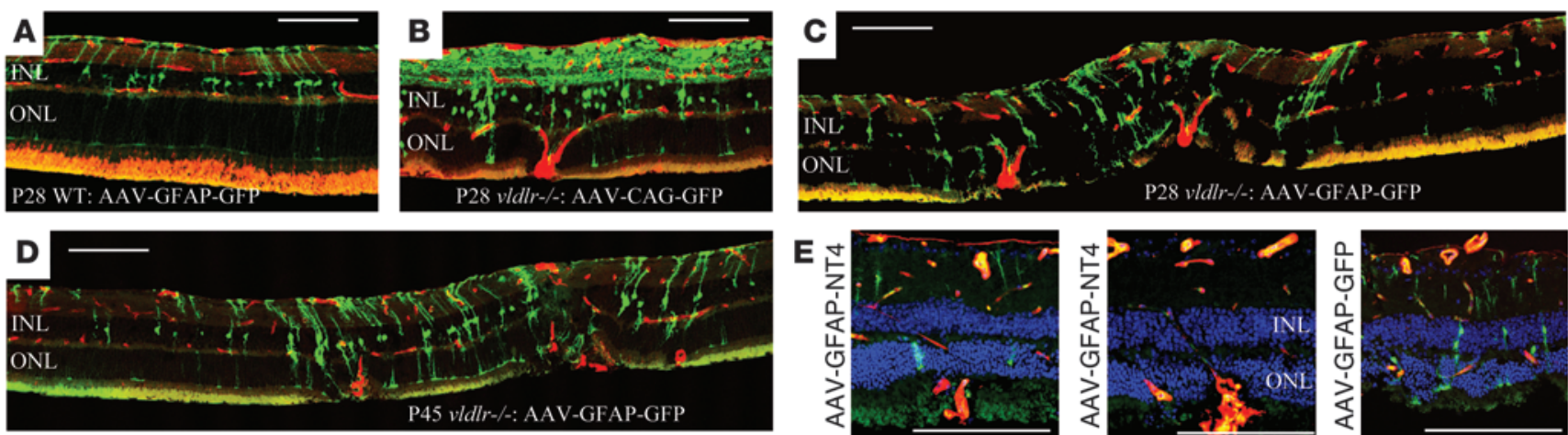

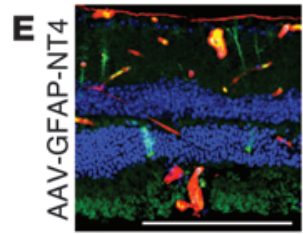

Goat $\alpha$ NT-4

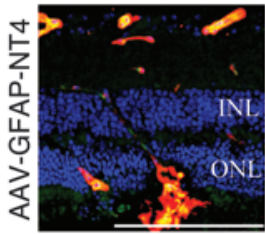

Goat IgG

Collagen IV (vessels), DAPI

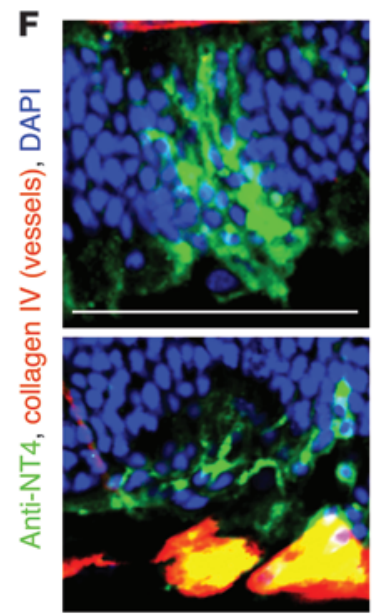

I AAV-GFAP-NT4
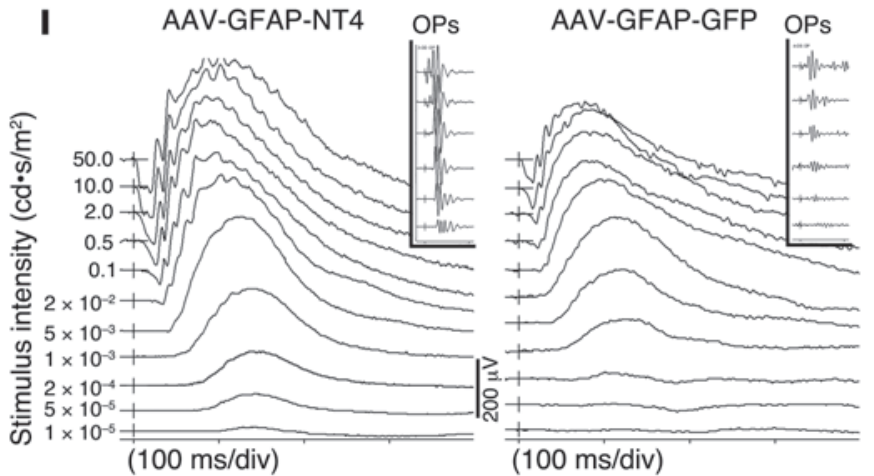

(100 ms/div)

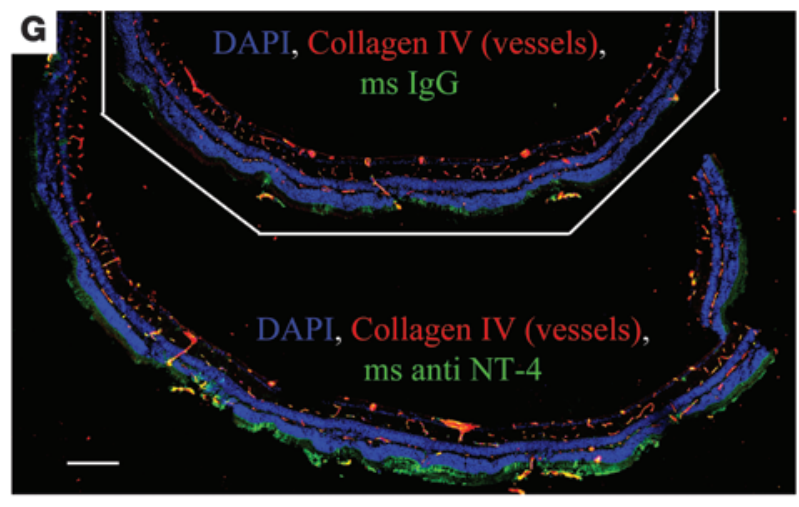

$\mathbf{H}$

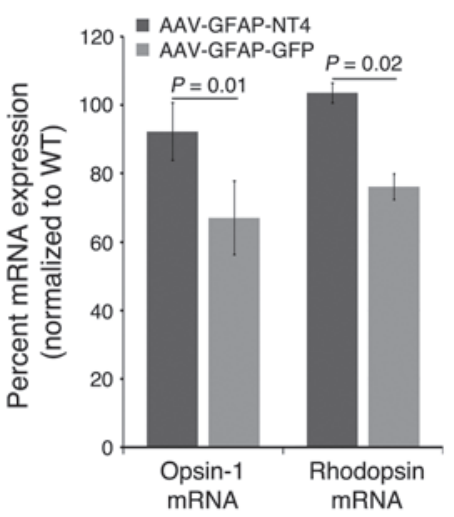

Figure 5

Targeted delivery of NT4 to sites of subretinal NV protects VIdlr ${ }^{-1-}$ retinas from neuronal degeneration. (A) AAV-GFAP-GFP-treated WT retinas exhibited minimal GFP expression in the inner retina and no expression in the outer retina. (B) AAV-CAG-GFP caused nonspecific GFP expression, mainly limited to the inner retina. (C and D) At 2 wk after intravitreal AAV-GFAP-GFP injection, GFP was observed in Müller glia specifically surrounding subretinal NV in P28 VIdlr ${ }^{-1-}$ mouse retinas (C), which was maintained at P45, 1 mo after injection (D). (E-G) NT4 gene product was produced in Müller cells adjacent to subretinal NV in AAV-GFAP-NT4-treated VIdlr-1- retinas (E and F), resulting in accumulation of NT4 at photoreceptor segments (G). Line in $\mathbf{G}$ separates control, pre-immune IgG-stained retina (top) from retina stained with anti-NT4 antibodies (bottom). (H) Quantitative RT-PCR analysis of opsin-1 and rhodopsin mRNA expression, normalized to age-matched WT controls, demonstrated protective effects of AAV-GFAP-NT4 treatment. Error bars denote SEM. (I and J) ERG analysis demonstrated that AAV-GFAP-NT4 treatment attenuated loss of retinal function. Scale bars: $100 \mu \mathrm{m}(\mathbf{A}-\mathbf{E}$ and $\mathbf{G}) ; 50 \mu \mathrm{m}(\mathbf{F})$.

95\% confidence intervals, the single exception being 9-cis-retinol dehydrogenase (Figure 6D and Supplemental Table 1). While not absolute, these data suggest that neuronal defects in $\mathrm{Vldll}^{-1-}$ mice are not caused by a generalized failure of oxidative stress defense and support the concept of a direct relationship between excessive NV, oxidative stress, and neuronal degeneration.
Antioxidant treatment protects against retinal damage in Vldlr- - mice. If oxidative stress is associated with neuronal damage in the $\mathrm{Vldlr}^{-/-}$ retina, we reasoned that treatment with commonly used antioxidants may attenuate this degeneration. Daily oral administration of an antioxidant cocktail consisting of ascorbic acid, $\alpha$-lipoic acid, and reduced L-glutathione for 6 weeks dramatically reduced the lev- 
Table 2

ERG measurements in 4-month-old WT and untreated, AAV-GFAP-GFP control-treated, and AAV-GFAP-NT4-treated VIdl/r-- mice 3.5 months after injection

\begin{tabular}{|c|c|c|c|c|c|c|}
\hline Stimulus & Measurement & $\begin{array}{c}\text { WT } \\
(n=12)\end{array}$ & $\begin{array}{c}\text { VIdIr-/- } \\
\text { untreated } \\
(n=10)\end{array}$ & $\begin{array}{c}\text { VIdIr-/- } \\
\text { GFP } \\
(n=15)\end{array}$ & $\begin{array}{c}\text { VIdIr-/- } \\
\text { NT4 } \\
(n=15)\end{array}$ & $\begin{array}{c}P \\
\text { (NT4 vs. GFP) }\end{array}$ \\
\hline \multicolumn{7}{|c|}{ Scotopic measurements ${ }^{A}$} \\
\hline Low intensityB & B-wave implicit time (ms) & $121 \pm 5$ & $130 \pm 4$ & $126 \pm 4$ & $118 \pm 2$ & $0.003^{H}$ \\
\hline High intensityc & A-wave amplitude $(\mu \mathrm{V})$ & $136 \pm 25$ & $80 \pm 17$ & $68 \pm 13$ & $114 \pm 18$ & $0.011^{H}$ \\
\hline High intensity & A-wave implicit time (ms) & $24 \pm 1$ & $24 \pm 1$ & $24 \pm 1$ & $22 \pm 1$ & 0.067 \\
\hline High intensity & B-wave implicit time (ms) & $80 \pm 4$ & $89 \pm 2$ & $93 \pm 4$ & $75 \pm 2$ & $0.0002^{\mathrm{H}}$ \\
\hline Maximum OPD & Amplitude $(\mu \mathrm{V})$ & $71 \pm 10$ & $39 \pm 7$ & $36 \pm 7$ & $62 \pm 12$ & $0.013^{H}$ \\
\hline \multicolumn{7}{|c|}{ Photopic measurements ${ }^{E}$} \\
\hline $\begin{array}{l}1 \mathrm{~Hz}^{\mathrm{F}} \\
1 \mathrm{~Hz} \\
1 \mathrm{~Hz}\end{array}$ & $\begin{array}{l}\text { A-wave implicit time (ms) } \\
\text { B-wave amplitude }(\mu \mathrm{V}) \\
\text { B-wave implicit time (ms) }\end{array}$ & $\begin{array}{r}19 \pm 2 \\
105 \pm 9 \\
62 \pm 4\end{array}$ & $\begin{array}{l}23 \pm 1 \\
83 \pm 6 \\
71 \pm 3\end{array}$ & $\begin{array}{l}22 \pm 1 \\
80 \pm 11 \\
78 \pm 3\end{array}$ & $\begin{array}{l}18 \pm 1 \\
96 \pm 9 \\
58 \pm 2\end{array}$ & $\begin{array}{l}0.005^{\mathrm{H}} \\
0.022^{\mathrm{H}} \\
0.0001^{\mathrm{H}}\end{array}$ \\
\hline $\begin{array}{l}16 \mathrm{~Hz} \text { flicker } \mathrm{G} \\
16 \mathrm{~Hz} \text { flicker }\end{array}$ & $\begin{array}{l}\text { Amplitude }(\mu \mathrm{V}) \\
\text { Implicit time }(\mathrm{ms})\end{array}$ & $\begin{array}{l}19 \pm 3 \\
55 \pm 3\end{array}$ & $\begin{array}{l}16 \pm 2 \\
58 \pm 1\end{array}$ & $\begin{array}{l}15 \pm 2 \\
58 \pm 1\end{array}$ & $\begin{array}{l}19 \pm 2 \\
56 \pm 1\end{array}$ & $\begin{array}{l}0.041^{H} \\
0.175\end{array}$ \\
\hline
\end{tabular}

Values are mean \pm SEM. ADark-adapted measurements performed to determine rod and mixed cone-rod function. ${ }^{\mathrm{B}} 0.005 \mathrm{~cd} \bullet \mathrm{s} / \mathrm{m}^{2}$. ${ }^{C_{2}} \mathrm{~cd} \cdot \mathrm{s} / \mathrm{m}^{2}$. ${ }^{\mathrm{D}} 10 \mathrm{~cd} \bullet \mathrm{s} / \mathrm{m}^{2}$. ELight-adapted measurements performed to determine cone function. $\mathrm{F}_{20} \mathrm{~cd} \bullet \mathrm{s} / \mathrm{m}^{2}$. $\mathrm{G}_{10} \mathrm{~cd} \bullet \mathrm{s} / \mathrm{m}^{2}$. HStatistically significant difference between contralaterally AAV-GFAP-NT4-and AAV-GFAP-GFP-treated eyes. tissue. At the other extreme, excessive vessels (NV) can also compromise tissue function, usually as a result of leakage, hemorrhage, or scarring caused by the abnormal new vessels. In this study, we provide evidence for yet another mechanism of injury associated with $\mathrm{NV}$, even in the absence of clinically significant leakage or hemorrhage: neuronal cell death as a result of increased oxidative stress caused by proximity to the abnormal vessels.

In several diseases that can cause irreversible vision loss, such as RAP (16) and MacTel $(14,41)$, abnormal vessels extend from the highly vascularized inner retina into the normally avascular outer retina. The retina of the $\mathrm{Vldlr}^{-/}$mouse exhibits vascular changes very similar to those observed in patients with MacTel and RAP and thus is a

els of oxidative stress in $\mathrm{Vldlr}^{-/-}$mouse retinas (Figure 7, A and B). This correlated with protection of the photoreceptors from degeneration. Furthermore, 6 weeks of antioxidant treatment initiated at 2 months of age, well after abnormal subretinal NV is established, significantly improved the expression levels of opsin and rhodopsin in the retinas of 3.5-month-old $\mathrm{Vldlr}^{-1-}$ mice (Figure 7C). Opsin and rhodopsin expression levels remained similar to those of untreated 2-month-old Vldlr ${ }^{-/-}$mice, which suggests that antioxidant treatment prevented further loss of cones and rods from the time treatment was initiated. Antioxidant treatment did not have any effect on the formation and persistence of subretinal NV (Figure 7, D and E). Thus, the observed activity was not caused by prevention or elimination of $\mathrm{NV}$, but rather by protection of the neurons from secondary neurodegeneration associated with NV. Normalized ERGs were also observed in $\mathrm{Vldlr}^{-/-}$mice on an antioxidant diet compared with vehicle-treated animals. Importantly, significant preservation of retinal function was observed even when treatments were initiated at 2 months of age, an age at which intra- and subretinal NV was well established and oxidative stress was already apparent (Figure 7, F and G, and Table 4), consistent with the results obtained from the RT-PCR experiments. ERGs of animals in which treatment was initiated at an earlier age were similar to those observed in WT animals (data not shown), which indicates that early dietary measures may provide nearly full protection from neuronal degeneration in the retina. The profound protective effect of antioxidant dietary supplementation on retinal degeneration further supports a direct link between oxidative stress and neuronal degeneration in the Vldlr ${ }^{-1}$ mouse retina.

\section{Discussion}

Paucity and dysfunction of blood vessels in tissues leading to ischemia is a well-recognized pathological process that often leads to irreversible injury, particularly in neuronal good model for studying the general relationship between $\mathrm{NV}$ and neurodegeneration. The new vessels observed in the human disease and the Vldl ${ }^{-1-}$ mouse exhibit relatively mild permeability defects and are accompanied by glial activation and disruption of the RPE. Another feature common to RAP, MacTel, and the Vldlr-/- retina is that the nonuniformly distributed focal vascular lesions are directly associated with neuronal degeneration separated by relatively normal regions. While other retinal degenerative models, such as $r d 1$ or $r d s$ mice, have associated vascular abnormalities and neuronal loss, many of these result in uniform degeneration and relate more closely to genetic diseases, such as retinitis pigmentosa, rather than to ocular vascular disease. Taken together, these similarities support the use of the $\mathrm{Vldlr}^{-/}$mouse as a compelling model to investigate selected human retinal diseases (e.g., MacTel) as well as other, nonocular neurodegenerative disorders with associated NV.

VEGF plays a critical role in normal retinal vascularization and retinal angiogenic diseases (42-44). In the Vldlr ${ }^{-/-}$retina, VEGF upregulation is observed in photoreceptors and RPE and partici-

\section{Table 3}

Metabolite analysis of 6-month-old WT and VIdlr-/-mouse retinas

\begin{tabular}{|c|c|c|c|c|}
\hline Metabolite & $\begin{array}{c}\text { Molecular } \\
\text { formula }\end{array}$ & $\begin{array}{c}m / z \\
\text { observed }\end{array}$ & $P$ & $\begin{array}{l}\text { VIdIr-1- fold } \\
\text { upregulation }\end{array}$ \\
\hline L-Carnitine & $\mathrm{C}_{7} \mathrm{H}_{15} \mathrm{NO}_{3}$ & 162.11 & $5.45 \times 10^{-6}$ & 2.58 \\
\hline L-Acetylcarnitine & $\mathrm{C}_{9} \mathrm{H}_{17} \mathrm{NO}_{4}$ & 204.12 & $1.03 \times 10^{-10}$ & 3.00 \\
\hline Isovalerylcarnitine & $\mathrm{C}_{12} \mathrm{H}_{23} \mathrm{NO}_{4}$ & 246.17 & $2.55 \times 10^{-6}$ & 2.28 \\
\hline Isohydrosorbylcarnitine & $\mathrm{C}_{13} \mathrm{H}_{23} \mathrm{NO}_{4}$ & 258.16 & $2.90 \times 10^{-10}$ & 3.27 \\
\hline Hexanoylcarnitine & $\mathrm{C}_{13} \mathrm{H}_{25} \mathrm{NO}_{4}$ & 260.18 & $1.58 \times 10^{-7}$ & 2.18 \\
\hline Hydroxyoctanoylcarnitine & $\mathrm{C}_{15} \mathrm{H}_{29} \mathrm{NO}_{4}$ & 304.21 & $6.66 \times 10^{-7}$ & 3.39 \\
\hline
\end{tabular}

Shown are metabolites with an increased presence in VIdlr-/- retinas, including several carnitine derivatives that are generally upregulated in response to increased oxidative stress. 
A
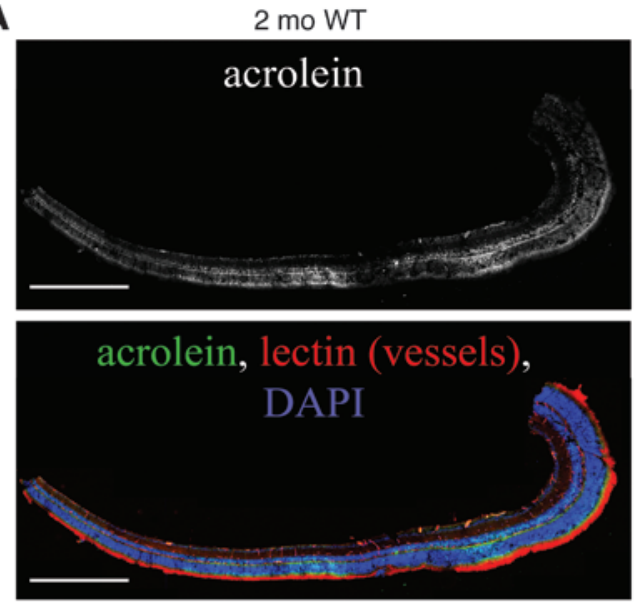

B
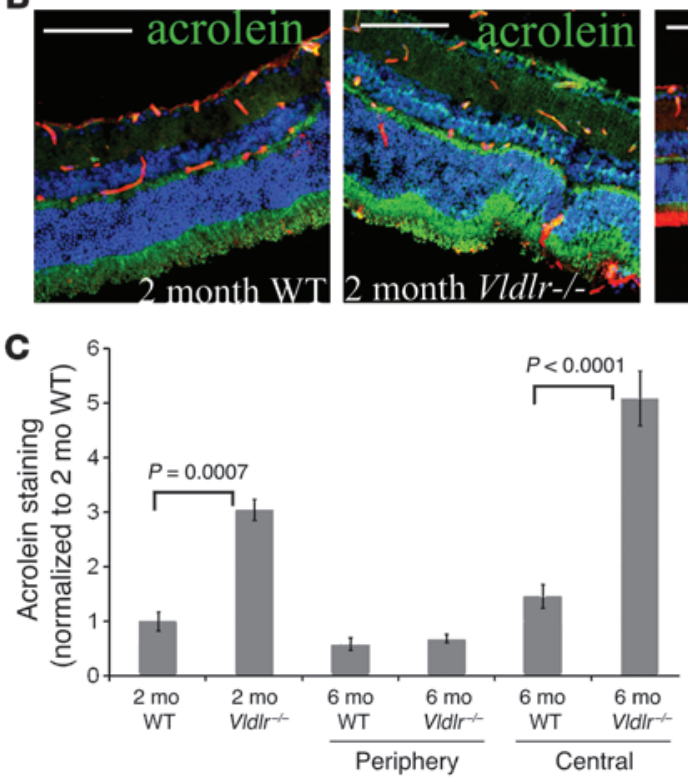

Figure 6

Evidence of oxidative stress associated with subretinal NV in VIdlr--- mouse retinas. (A) Acrolein staining increased in VIdlr $r^{-1}$ retinas compared with age-matched WT controls. Acrolein staining in $\mathrm{VIdll}^{-1-}$ retinas was largely within the central retina, where intraretinal NV is most prominent. Panels are composite montages of multiple serial micrographs. (B) Higher-magnification images demonstrating that acrolein staining localized to the photoreceptor layer and INL. (C) Acrolein staining significantly increased in 2-mo-old VIdlr ${ }^{-1-}$ compared with WT retinas. At 6 mo of age, acrolein staining was similar in the peripheral retinas

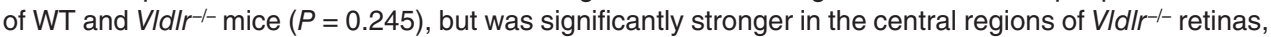
where subretinal NV occurs, compared with retinas of WT mice. Error bars denote SEM. (D) Expression of genes involved in oxidative stress defense mechanisms was similar between P21 $\mathrm{VIdll}^{-1-}$ and WT retinas. Solid line indicates equivalent expression levels; dotted lines represent $95 \%$ confidence level ranges. Scale bars: $500 \mu \mathrm{m}(\mathbf{A}) ; 50 \mu \mathrm{m}(\mathbf{B})$.

pates in the stimulation of subretinal NV. Normal VLDLR expression within the avascular outer retina, and upregulation of VEGF coincident with formation of abnormal subretinal vessels in these areas of VLDLR-deficient mice, suggests a mechanism linking VLDLR with proangiogenic factors. No obvious abnormalities were observed in the choroid, suggesting potential RPE polarity of the VEGF overexpression toward the inner retina. VLDLR is a negative regulator of wnt signaling during retina development; upregulation of the wnt coreceptor LRP5/6 and increased down- stream wnt signaling occurs in Vldlr $^{-1-}$ mouse retinas (27). VEGF is a well-known product of wnt signaling, and VLDLR may normally participate in turning off wnt signaling once retinal vascular development is complete. In the absence of VLDLR, wnt signaling persists, leading to increased levels of VEGF expression in photoreceptor and RPE layers and the angiogenic extension of abnormal intra- and subretinal vessels. Whether such a mechanism operates in diseases such as MacTel and RAP is uncertain, although anti-VEGF therapy has previously been tested in both conditions $(45,46)$. Initial efforts to treat RAP lesions using combination angiostatics are also promising (47). However, in our experience with the $V l d l r^{-/-}$ mouse, antiangiogenic therapy is only transiently effective, subretinal NV returns within 2 weeks, and therapy must be initiated prior to NV formation. Thus, the usefulness of antiangiogenic therapy in human ocular diseases with attributes similar to those of Vldlr ${ }^{-/-}$mice may be limited.

A major finding in this study is that neuronal abnormalities and impaired retinal function developed in association with NV. We were able to prevent focal degeneration of photoreceptors in the regions of vascular abnormalities through the selective delivery of neurotrophic molecules by taking advantage of cellular changes observed in this condition. Müller glia, with cytoplasmic processes spanning the inner and outer retina, were activated in response to vascular changes such as those observed in the Vldlr $^{-/-}$retina. Upon activation, these cells upregulate GFAP, and their appearance and location precisely correlate, both temporally and spatially, with subretinal NV and associated neuronal degeneration in the outer retina. By targeting the activated Müller cells and taking advantage of their retina-spanning cytoplasmic processes, NT4 was specifically delivered to sites of vascular abnormalities after intravitreal injection of AAV-GFAP-NT4. This strategy using endogenous cells (e.g., activated Müller cells) to deliver gene therapy products to the outer retina could be useful clinically to avoid the need for subretinal injection of the viral vector, a procedure that can have deleterious effects on already diseased 
A

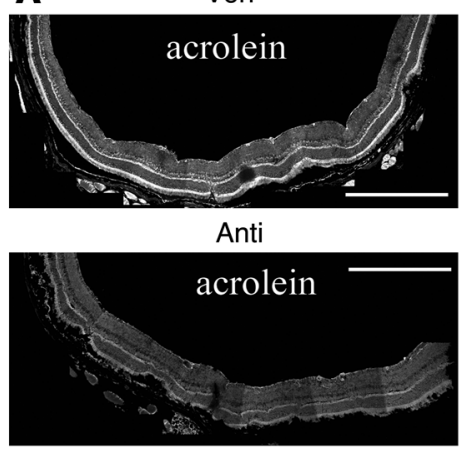

D
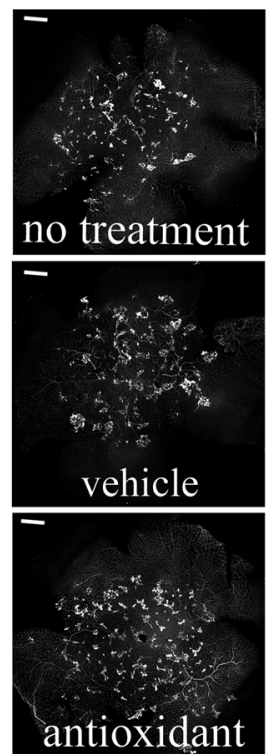

B

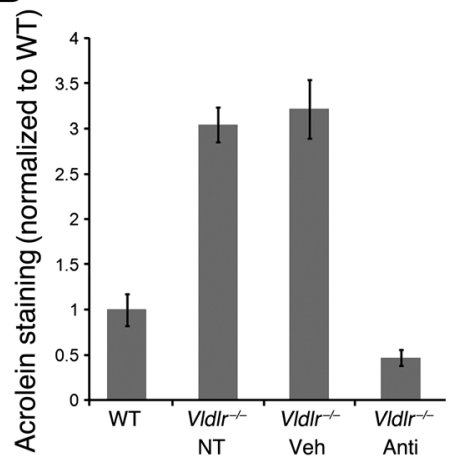

F
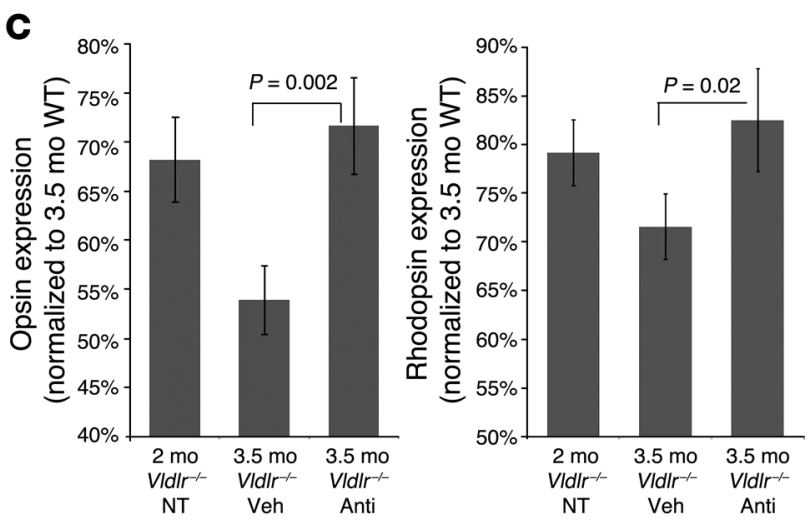

E

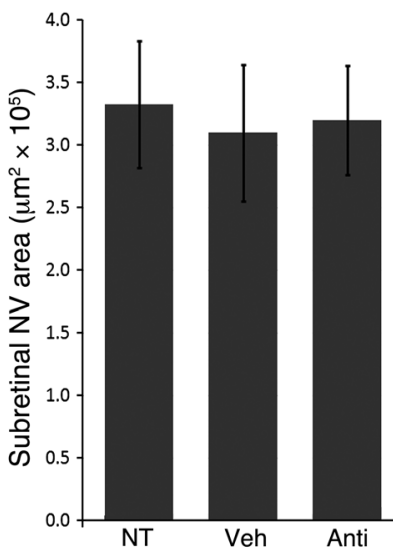

Veh
Anti

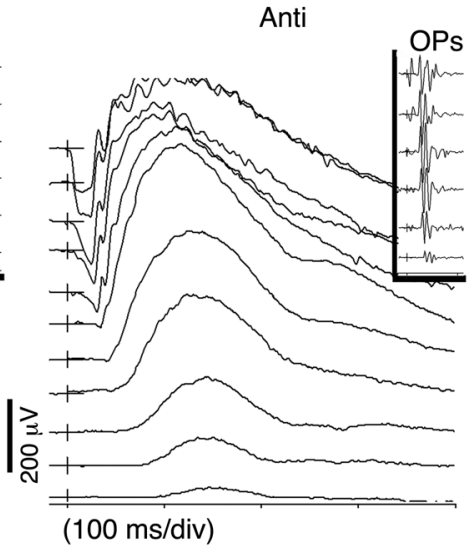

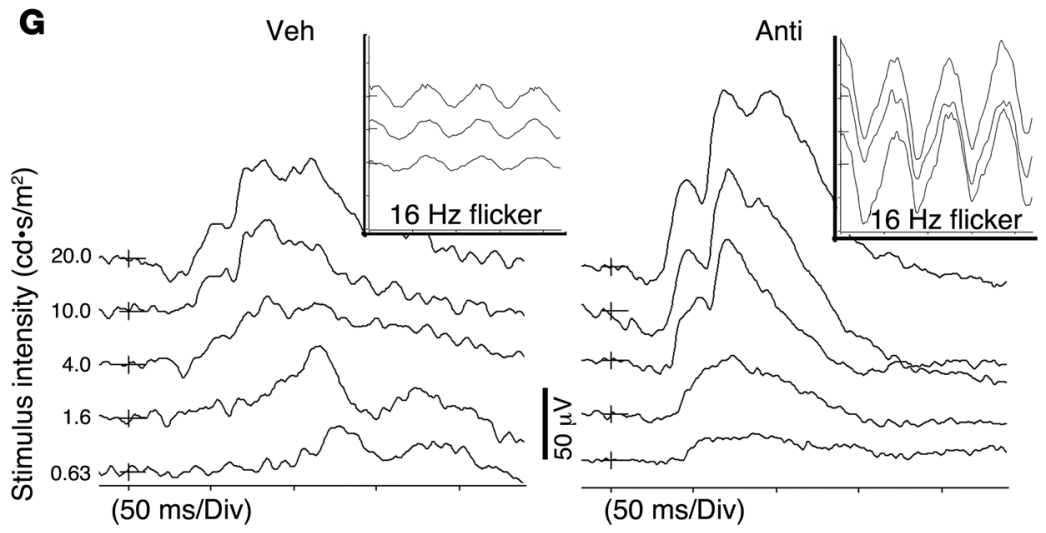

Figure 7

Antioxidants protect retinas from degeneration and reduced function in VIdl/r-/- retinas. (A and B) In retinas of antioxidant-treated 2-mo-old

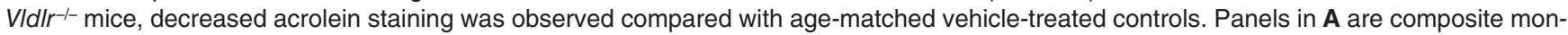
tages of multiple serial micrographs. Error bars denote SEM. NT, not treated; Anti, antioxidant treatment. (C-G) VIdlr ${ }^{-/-}$mice were subjected to a 6-wk treatment with vehicle or antioxidants, beginning at 2 mo of age. (C) Antioxidant treatment attenuated the loss of cones and rods, as demonstrated by normalized expression of opsin-1 and rhodopsin, respectively. $n=12$ retinas per group. Error bars denote SEM. (D and E) The extent of subretinal NV in 3.5-mo-old VIdIr ${ }^{-1-}$ mouse retinas was not affected by antioxidant treatment. $n=12$ retinas per group. Error bars denote SEM. (F and G) ERGs were normalized after 6 wk of antioxidant treatment. Note decreased and delayed signaling in vehicle-treated VIdll ${ }^{-1-}$ mice compared with those treated with antioxidants. Scale bars: $250 \mu \mathrm{m}$.

retinas. In addition, we were able to dramatically attenuate retinal degeneration in the Vldlr/- mouse retina, supporting a link between the presence of subretinal vessels and neuronal degeneration.

$V_{l d l r^{-/}}$retinas exhibit increased levels of oxidative stress $(35,37$, 38 ). Excessive oxygen levels can generate reactive oxygen species that cause molecular damage to lipids, proteins, and nucleic acids, which can subsequently lead to cell death unless neutralized by the antioxidant defense system (48). The retina, with its high fat content and its exposure to light, is particularly susceptible to such injury. Cone loss is associated with oxidative damage in other models of primary retinal degeneration, such as the $r d 1$ mouse $(9,10)$, and oxidative damage has been linked with AMD (40). Recent studies using adaptive optics 
Table 4

ERG measurements of antioxidant- and vehicle-treated VIdIr-/- mice

\begin{tabular}{llrrl} 
Stimulus & Measurement & $\begin{array}{r}\text { Vehicle } \\
(\boldsymbol{n}=\mathbf{1 6})\end{array}$ & $\begin{array}{c}\text { Antioxidant } \\
(\boldsymbol{n}=\mathbf{1 8})\end{array}$ & $\boldsymbol{P}$ \\
$\begin{array}{l}\text { Scotopic measurements } \\
\text { Low intensityB }\end{array}$ & B-wave implicit time (ms) & $152 \pm 4$ & $142 \pm 4$ & $0.036^{\mathrm{H}}$ \\
High intensityC & A-wave amplitude $(\mu \mathrm{V})$ & $46 \pm 6$ & $112 \pm 12$ & $0.0001^{\mathrm{H}}$ \\
High intensity & A-wave implicit time (ms) & $30 \pm 1$ & $29 \pm 1$ & 0.321 \\
High intensity & B-wave implicit time (ms) & $111 \pm 5$ & $94 \pm 2$ & $0.001^{\mathrm{H}}$ \\
Maximum OPD & Amplitude $(\mu \mathrm{V})$ & $18 \pm 2$ & $30 \pm 3$ & $0.0047^{\mathrm{H}}$ \\
Photopic measurements & & & \\
$1 \mathrm{~Hz}$ & A-wave implicit time $(\mathrm{ms})$ & $28 \pm 1$ & $26 \pm 1$ & 0.051 \\
$1 \mathrm{~Hz}$ & B-wave amplitude $(\mu \mathrm{V})$ & $73 \pm 9$ & $114 \pm 6$ & $0.0003^{\mathrm{H}}$ \\
$1 \mathrm{~Hz}$ & B-wave implicit time (ms) & $88 \pm 3$ & $81 \pm 2$ & $0.041^{\mathrm{H}}$ \\
$16 \mathrm{~Hz}$ flickerG & Amplitude $(\mu \mathrm{V})$ & $12 \pm 1$ & $22 \pm 2$ & $0.0001^{\mathrm{H}}$ \\
$16 \mathrm{~Hz}$ flicker & Implicit time $(\mathrm{ms})$ & $68 \pm 2$ & $71 \pm 2$ & 0.12 \\
\hline
\end{tabular}

Values are mean $\pm \mathrm{SEM}$. ${ }^{A}$ Dark-adapted measurements performed to determine rod and mixed cone-rod function. ${ }^{\mathrm{B}} 0.005 \mathrm{~cd} \cdot \mathrm{s} / \mathrm{m}^{2}$. ${ }^{\mathrm{C}} 2 \mathrm{~cd} \bullet \mathrm{s} / \mathrm{m}^{2}$. ${ }^{\mathrm{D}} 10 \mathrm{~cd} \cdot \mathrm{s} / \mathrm{m}^{2}$. ELightadapted measurements performed to determine cone function. $\mathrm{F} 20 \mathrm{~cd} \bullet \mathrm{s} / \mathrm{m}^{2}$. $\mathrm{G}_{10} \mathrm{~cd} \bullet \mathrm{s} / \mathrm{m}^{2}$. HStatistically significant difference between antioxidant- and vehicletreated $\mathrm{Vldll}^{-/-}$mouse retinas.

also demonstrate focal photoreceptor loss associated with vascular abnormalities in diseases such as MacTel, traditionally considered a retinal vascular disease (A. Roorda, unpublished observations). We attempted to directly assess the role of abnormal NV in causing subsequent neuronal degeneration by blocking $\mathrm{NV}$ in the $\mathrm{Vldlr} /-$ mice. However, even the most potent angiostatic combinations were only transiently effective, while the neurodegeneration was progressive over the course of several weeks to months after initiation of subretinal NV. Thus, prolonged elimination of subretinal NV was impossible without repeated injections, a process which at best leads to injury and variability in the small mouse eye, and often leads to phthisis. However, based on the strong association between subretinal $\mathrm{NV}$ and neurodegeneration, the correlation between subretinal $\mathrm{NV}$ and oxidative stress, and the neuroprotective results obtained from treatment with antioxidants, we hypothesize that increased vascularization and abnormal localization of the subretinal NV leads to increased oxidative stress and subsequent neuronal damage. No defects were found in the cellular oxidative stress defense pathways of the $\mathrm{Vldlr}^{-1}$ mice, further supporting our hypothesis.

These findings demonstrating protective effects of antioxidant treatment on neuronal degeneration associated with abnormal $\mathrm{NV}$ support prior epidemiological studies in humans showing that an antioxidant-rich diet protects against $\mathrm{AMD}(40)$ and diminishes its progression (49). However, it should be noted that only moderate levels of protection were observed in these epidemiological studies, particularly when dietary supplementation is initiated after NV is already present. Such dietary measures may be a simple and effective method of preventing neuronal cell loss associated with certain retinal vascular diseases such as MacTel and RAP. In instances of associated neuronal degeneration with substantial ERG abnormalities, gene therapy using intravitreally injected AAV-2 vectors encoding neurotrophic transgenes may also serve to preserve visual function. These findings using the $\mathrm{Vldl}^{-1-}$ mouse retina model also support other studies that demonstrate benefits of antioxidant-rich diets in relation to other neurodegenerative disorders of the CNS (50). Thus, our present findings may also have application to other neurodegenerative disorders, such as Huntington disease, Parkinson disease, and Alzheimer disease. Further studies will be required to better understand the link between vascular abnormalities such as those observed in retinal $\mathrm{NV}$, oxidative stress, and neuronal degeneration.

\section{Methods \\ Retina dissection and staining. All animal studies were approved by the animal protocols review committee at The Scripps Research Institute. Vldlr-/- mice $(23,51)$ were obtained and backcrossed in the C57BL/6J background for at least 10 generations. WT mice were normal, nonknockout C57BL/6J mice. Retinas were dis- sected and prepared for whole mounts or sectioning as previously described (17). For preparation of retinal cross-sections, dissected retinas were laid flat with 4 radial relaxing incisions, placed in $4 \%$ $\mathrm{PFA}$, and incubated at $4^{\circ} \mathrm{C}$ overnight. Retinas were then placed in $20 \%$ sucrose at $4^{\circ} \mathrm{C}$ for 4 hours and embedded in Tissue-Tek OCT compound (Sakura FineTechnical) for cryosectioning. \\ Fluorescently conjugated isolectin GS from Griffonia simplici- folia (Invitrogen) or antibodies against collagen IV (Chemicon), GFAP (DAKO), and red/green opsin (Chemicon) were used for immunohistochemistry. For analysis of vascular leak, FITC- dextran (43,200 MW; Sigma-Aldrich) was perfused through the left ventricle of deeply anesthetized mice using $150 \mu \mathrm{l}$ of} $50 \mathrm{mg} / \mathrm{ml}$ solution in PBS.

Electron microscopy analysis of Vldlr ${ }^{-1}$ retinas. Eye cups containing the retina and choroid from each mouse were fixed in $4 \%$ paraformaldehyde plus $1.5 \%$ glutaraldehyde in $0.1 \mathrm{M}$ cacodylate buffer overnight at $4{ }^{\circ} \mathrm{C}$ followed by rinsing in $0.1 \mathrm{M} \mathrm{Na}$ cacodylate buffer for 1 hour. The eye cups were then postfixed in $1 \% \mathrm{OsO}_{4}$ in $0.1 \mathrm{M}$ cacodylate buffer for 2 hours, followed by another 1-hour wash and dehydration with graded ethanol solutions. Samples were incubated overnight in a 1:2 mixture of propylene oxide and Epon/Araldite (Sigma-Aldrich) and then placed in 100\% resin followed by embedding. The blocks were sectioned and used for high-magnification electron microscopy analysis.

Laser capture. All steps were carefully performed using RNAse-free conditions. Whole eyes were isolated without fixation and embedded in OCT. After serial sectioning, laser capture microdissection was performed using an Arcturus PixCell II Laser Capture Microdissection microscope. The ganglion cell layer, INL, ONL, photoreceptor inner/outer segments, and retinal pigment epithelium were isolated separately from histologically stained retinas. Segments highly enriched for vessel fragments were also isolated after a brief stain with isolectin GS (Invitrogen). RNA was isolated from the microdissected retina using microRNA kits (Qiagen) and, after ensuring the quality of each RNA preparation, was reverse transcribed into cDNA for analysis by quantitative RT-PCR.

Quantitative RT-PCR analysis. Retinas were dissected under RNAse free conditions, and each retina was immediately lysed in separate tubes containing RLTplus buffer (Qiagen) with $\beta$-mercaptoethanol (Sigma-Aldrich). RNA was isolated using the RNeasy plus mini kit (Qiagen) protocol, which incorporates a genomic DNA elimination step. Total RNA $(0.5 \mu \mathrm{g}$ from each retina) was reverse transcribed into cDNA using the Quantitect reverse transcription kit (Qiagen). SYBR green-based real-time quantitative RT-PCR was performed using the iCycler machine (BioRad) to determine mRNA expression levels of various gene products. Expression levels from each retina were normalized to $\beta$-actin.

Primers. The following primers were used: cone opsin forward, $5^{\prime}$ CAAGCCCTTTGGCAATGTGA-3'; cone opsin reverse, 5'-GCTCCAACCAAAGATTGGTGG-3'; rhodopsin forward, 5'-TCATGGTCTTCGGAGGATTCAC-3'; rhodopsin reverse, 5'-TCACCTCCAAGTGTGGCAAAG-3'; 
VLDLR forward, 5'-TTCCTAGCTCATCCTCTTGCAC-3'; VLDLR reverse, 5'-CTGACCCAGTGAATTTATTGGC-3'; VEGF forward, 5'-GGAGACTCTTCGAGGAGCACTT-3'; VEGF reverse, 5'-GGCGATTTAGCAGCAGATATAAGAA-3'. For $\beta$-actin normalization, the Mm_Actb_2_SG QuantiTect Primer Assay was used (Qiagen).

Treatment of murine eyes with antiangiogenics. Intravitreal injections of Macugen, combination angiostatics, or vehicle controls were performed at P12, just prior to formation of the subretinal vascular sprouts in the $\mathrm{Vldl}{ }^{-/-}$retinas. All intravitreal injections were performed using a Hamilton syringe fitted with a 33-gauge needle (Hamilton), injecting $0.5 \mu \mathrm{l}$ of solutions containing $1.5 \mu \mathrm{g}$ Macugen $(3.0 \mathrm{mg} / \mathrm{ml}$; Pfizer), a triple angiostatic combination, or PBS vehicle. The triple combination solution consisted of Macugen $(3.0 \mathrm{mg} / \mathrm{ml})$, integrin $\alpha_{v} \beta_{3}$ and $\alpha_{v} \beta_{5}$ antagonist EMD472523 $(20 \mathrm{mg} / \mathrm{ml}$; Merck $)$, and T2$\operatorname{TrpRS}(0.5 \mathrm{mg} / \mathrm{ml}$; Angiosyn). T2-TrpRS is a fragment of human TrpRS that has previously been shown to have angiostatic activity (30). The retinas were analyzed 1 week later at P20 for the formation of subretinal vessels. Wholemount preparations were stained for the vasculature, the subretinal space was imaged using confocal microscopy, and the number of subretinal vascular sprouts as well as the area of subretinal NV staining was quantified.

ERG. After overnight dark adaptation, mice were anesthetized under dim red light by intraperitoneal injection of $15 \mathrm{mg} / \mathrm{kg}$ ketamine and $7 \mathrm{mg} / \mathrm{kg}$ xylazine. Full-field ERGs were recorded from the corneal surface of each eye after pupil dilation ( $1 \%$ tropicamide and $2.5 \%$ phenylephrine) using a gold loop electrode together with a mouth reference and tail ground electrode, similar to previously published methods (52). A computerized system with an electronically controlled Ganzfeld dome was used (Espion E2 with Colordome; Diagnosys). In the dark-adapted state, we recorded rod and mixed cone/rod responses to a series of white flashes of increasing intensities $\left(1 \times 10^{-5}\right.$ to $\left.50 \mathrm{~cd} \bullet \mathrm{s} / \mathrm{m}^{2}\right)$. In the light-adapted state, with a 30 $\mathrm{cd} / \mathrm{m}^{2}$ background, cone responses to $1-\mathrm{Hz}\left(0.63\right.$ to $\left.20 \mathrm{~cd} \bullet \mathrm{s} / \mathrm{m}^{2}\right)$ and $30-\mathrm{Hz}$ $\left(3.98,10\right.$, and $\left.20 \mathrm{~cd} \bullet \mathrm{s} / \mathrm{m}^{2}\right)$ flicker stimuli were recorded. All ERG responses were filtered at $0.3-500 \mathrm{~Hz}$, and signal averaging was applied.

$A A V-2$ vector constructs. The AAV-2 vector genome backbone consisting of AAV-2 inverted terminal repeats flanking a neurotrophic factor transgene expression cassette with the ubiquitous CAG promoter and the human growth hormone gene polyadenylation signal (Stratagene) was described previously (53). The construction of the AAV-GFAP-NT4 vector genome was performed in 2 steps. First, human NT4 cDNA was isolated from a human cDNA library (Clontech) by PCR and inserted into the AAV-CAG-Neurturin (NTN) in place of the NTN cDNA (53) to generate AAV-CAG-NT4. Second, a minimal 0.35-kb glial cell-specific GFAP promoter consisting of the A/B (-1757 to -1488$)$ and $D(-132$ to -56$)$ sequences of the human GFAP promoter (54) was isolated by PCR from human genomic DNA (Novagen), spliced together, and inserted into AAV-CAG-NT4 in place of the CMV enhancer and chicken $\beta$-actin promoter elements of the CAG promoter. Plasmid clone identities were confirmed by restriction digestions and nucleotide sequence determination.

Cell culture and AAV-2 vector production. AAV-2 vectors were produced by standard triple plasmid transfection technique in human embryonic kidney 293 cells and purified by multiple chromatography and filtration steps as described previously (53). After purification, vectors were resuspended in formulation buffer (PBS plus $2 \mathrm{mM} \mathrm{MgCl}_{2}$ ), and vector titer was determined by quantitative PCR and expressed as vector genomes $/ \mathrm{ml}$.

Treatment of murine eyes with AAV-2 vectors. Solutions $(0.5 \mu \mathrm{l})$ of AAV- 2 particles containing AAV-CAG-GFP (CAG promoter-driven GFP expression), AAV-GFAP-GFP (GFAP promoter-driven GFP expression), or AAV-GFAPNT4 (GFAP promoter-driven NT4 expression), with titers of approximately $1 \times 10^{13}$ vector genomes $/ \mathrm{ml}$, were injected intravitreally into the eyes of 2-week-old $\mathrm{Vldlr}^{-1-}$ mice. Viral transfection was assessed by analyzing GFP expression at 1 and 2 months after injection. Effects on the neurodegenerative phenotype of $\mathrm{Vldlr}^{-1-}$ mice were assessed 3 months after injection.
Acrolein staining for oxidative stress. Retinas were stained as described above, either with antibodies against acrolein (1:200 dilution, catalog no. ab37110; Abcam) or with appropriate control IgGs. After staining was complete, all retinas were imaged using confocal microscopy using identical settings, including laser power, iris opening, and detector sensitivity. Thus, differences in intensity correlated directly to differences in the amount of acrolein present. A baseline staining threshold level was established, and corresponding images from retinal sections within the central two-thirds or retinal periphery were quantified by comparing areas of acrolein staining above this threshold.

Metabolite extraction and mass spectrometry analysis. Proteins were precipitated and metabolites extracted from $12 \mathrm{Vldlr}^{-/}$and $12 \mathrm{WT}$ retinas. After removal of proteins and isolation of metabolites, specimens were subjected to reversed phase and hydrophilic interaction chromatography. Metabolites with differential concentrations in $\mathrm{Vldl}^{-/}$- compared with age-matched WT retinas were identified by exact mass $(m / z)$ using METLIN (http://metlin.scripps. edu/), Human Metabolome Project (http://metabolomics.ca/), Lipid Maps (http://www.lipidmaps.org/), Biological Magnetic Resonance Data Bank (http://www.bmrb.wisc.edu/), and KEGG ligand (http://www.genome.jp/ kegg/ligand.html) databases. For a complete description of the materials and methods used for metabolomics analysis, see Supplemental Methods.

Microarray analysis. RNA was isolated from 3 sets of pooled retinas (6 retinas per set) from 3-week-old WT or Vldlr/- mice, yielding triplicate samples accounting for both biological and technical variance. Samples were prepared and hybridized to Mu74Av2 gene chips (Affymetrix) according to the manufacturer's instructions, and data were analyzed using GeneSpring software (Silicon Genetics). Genes related to oxidative stress pathways were analyzed for retinal expression, and only those above background in WT or Vldlr ${ }^{-/-}$retinas with statistically consistent results amongst the replicates were analyzed further. The expression levels were compared between the WT and $\mathrm{Vldl}^{--}$groups for each individual gene. Raw data values obtained from the Affymetrix analysis are included in Supplemental Table 1.

Antioxidant treatment. Littermates of $\mathrm{Vldl}^{-/}$mice were randomly separated into control and treated groups. Treated groups were fed a mixture of antioxidants that included $200 \mathrm{mg} / \mathrm{kg}$ ascorbic acid, $20 \mathrm{mg} / \mathrm{kg} \alpha$-lipoic acid, and $10 \mathrm{mg} / \mathrm{kg}$ L-glutathione (Sigma-Aldrich) in 1\% aqueous sucrose. Controls were fed vehicle consisting of $1 \%$ aqueous sucrose. All mice were fed by orally pipetting $10-30 \mu \mathrm{l}$ of solution and monitoring swallowing. Daily feedings were initiated at 2 months of age and were continued for 6 weeks.

Statistics. All data shown are mean \pm SEM. All $P$ values reported were derived using a standard Student's $t$ test with a 1-tailed distribution, and 2 sample equal variance for treatment groups using unpaired eyes or paired values for studies that used contralateral eyes to compare various treatments. A $P$ value less than 0.05 was considered significant.

\section{Acknowledgments}

We thank Malcolm Wood for technical assistance with electron microscopy and Christopher Aderman, Kip Conner, and Lois Smith (Harvard Medical School, Boston, Massachusetts, USA) for assistance with laser capture microdissection. This work was funded by National Eye Institute, NIH, grant R01 EY011254 (to M. Friedlander), by the MacTel Foundation, and by the V. Kann Rasmussen Foundation. M.I. Dorrell is supported by a fellowship from the California Institute for Regenerative Medicine.

Received for publication June 30, 2008, and accepted in revised form December 10, 2008.

Address correspondence to: Martin Friedlander, Department of Cell Biology, The Scripps Research Institute, MB-26, 10550 N. Torrey Pines Road, La Jolla, California 92037, USA. Phone: (858) 784-9138; Fax: (858) 784-9135; E-mail: friedlan@scripps.edu. 
1. Barcia, C., Emborg, M.E., Hirsch, E.C., and Herrero, M.T. 2004. Blood vessels and parkinsonism. Front. Biosci. 9:277-282.

2. Browne, S.E., and Beal, M.F. 2006. Oxidative damage in Huntington's disease pathogenesis. Antioxid. Redox Signal. 8:2061-2073.

3. Nunomura, A., et al. 2006. Involvement of oxidative stress in Alzheimer disease. J. Neuropathol. Exp. Neurol. 65:631-641.

4. Zhu, X., et al. 2007. Vascular oxidative stress in Alzheimer disease. J. Neurol. Sci. 257:240-246.

5. Faucheux, B.A., Bonnet, A.M., Agid, Y., and Hirsch, E.C. 1999. Blood vessels change in the mesencephalon of patients with Parkinson's disease. Lancet. 353:981-982.

6. Weinstein, B.M. 2005. Vessels and nerves: marching to the same tune. Cell. 120:299-302.

7. Tilleux, S., and Hermans, E. 2007. Neuroinflammation and regulation of glial glutamate uptake in neurological disorders. J. Neurosci. Res. 85:2059-2070.

8. Liu, P.K. 2003. Ischemia-reperfusion-related repair deficit after oxidative stress: implications of faulty transcripts in neuronal sensitivity after brain injury. J. Biomed. Sci. 10:4-13.

9. Shen, J., et al. 2005. Oxidative damage is a potential cause of cone cell death in retinitis pigmentosa. J. Cell. Physiol. 203:457-464.

10. Komeima, K., Rogers, B.S., Lu, L., and Campochiaro, P.A. 2006. Antioxidants reduce cone cell death in a model of retinitis pigmentosa. Proc. Natl. Acad. Sci. U. S. A. 103:11300-11305.

11. Klein, R., Peto, T., Bird, A., and Vannewkirk, M.R. 2004. The epidemiology of age-related macular degeneration. Am. J. Ophthalmol. 137:486-495.

12. Chew, E., Gillies, M., and Bird, A. 2006. Macular telangiectasia: a simplified classification. Arch. Ophthalmol. 124:573-574.

13. Gass, J.D. 2000. Histopathologic study of presumed parafoveal telangiectasis. Retina. 20:226-227.

14. Yannuzzi, L.A., et al. 2006. Idiopathic macular telangiectasia. Arch. Ophthalmol. 124:450-460.

15. Das, A., Puklin, J.E., Frank, R.N., and Zhang, N.L. 1992. Ultrastructural immunocytochemistry of subretinal neovascular membranes in age-related macular degeneration. Ophthalmology. 99:1368-1376.

16. Yannuzzi, L.A., et al. 2001. Retinal angiomatous proliferation in age-related macular degeneration. Retina. 21:416-434.

17. Dorrell, M.I., Aguilar, E., and Friedlander, M. 2002. Retinal vascular development is mediated by endothelial filopodia, a preexisting astrocytic template and specific R-cadherin adhesion. Invest. Ophthalmol. Vis. Sci. 43:3500-3510.

18. Dorrell, M.I., et al. 2004. Adult bone marrowderived stem cells utilize R-cadherin to target sites of neovascularization in the developing retina. Blood. 103:3420-3427.

19. Ohno-Matsui, K., et al. 2002. Inducible expression of vascular endothelial growth factor in adult mice causes severe proliferative retinopathy and retinal detachment. Am. J. Pathol. 160:711-719.

20. Heckenlively, J.R., et al. 2003. Mouse model of subretinal neovascularization with choroidal anastomosis. Retina. 23:518-522.

21. Li, C., et al. 2007. Biochemical alterations in the retinas of very low-density lipoprotein receptor knockout mice: an animal model of retinal angiomatous proliferation. Arch. Ophthalmol. 125:795-803.
22. Hu, W., et al. 2008. Expression of VLDLR in the retina and evolution of subretinal neovascularization in the knockout mouse model's retinal angiomatous proliferation. Invest. Ophthalmol. Vis. Sci. 49:407-415.

23. Frykman, P.K., Brown, M.S., Yamamoto, T., Goldstein, J.L., and Herz, J. 1995. Normal plasma lipoproteins and fertility in gene-targeted mice homozygous for a disruption in the gene encoding very low density lipoprotein receptor. Proc. Natl. Acad. Sci. U. S. A. 92:8453-8457.

24. Stone, J., and Dreher, Z. 1987. Relationship between astrocytes, ganglion cells and vasculature of the retina. J. Comp. Neurol. 255:35-49.

25. Fruttiger, M., et al. 1996. PDGF mediates a neuronastrocyte interaction in the developing retina. Newron. 17:1117-1131.

26. Erickson, K.K., Sundstrom, J.M., and Antonetti, D.A. 2007. Vascular permeability in ocular disease and the role of tight junctions. Angiogenesis. 10:103-117.

27. Chen, Y., Hu, Y., Lu, K., Flannery, J.G., and Ma, J.X. 2007. Very low density lipoprotein receptor, a negative regulator of the wnt signaling pathway and choroidal neovascularization. J. Biol. Chem. 282:34420-34428.

28. Dorrell, M.I., et al. 2007. Combination angiostatic therapy completely inhibits ocular and tumor angiogenesis. Proc. Natl. Acad. Sci. U. S. A. 104:967-972.

29. Banin, E., et al. 2006. T2-TrpRS inhibits preretinal neovascularization and enhances physiological vascular regrowth in OIR as assessed by a new method of quantification. Invest. Ophthalmol. Vis. Sci. 47:2125-2134.

30. Otani, A., et al. 2002. A fragment of human TrpRS as a potent antagonist of ocular angiogenesis. Proc. Natl. Acad. Sci. U. S. A. 99:178-183.

31. Tzima, E., et al. 2005. VE-cadherin links tRNA synthetase cytokine to anti-angiogenic function. J. Biol. Chem. 280:2405-2408.

32. Bradley, J., Ju, M., and Robinson, G.S. 2007. Combination therapy for the treatment of ocular neovascularization. Angiogenesis. 10:141-148.

33. Lykissas, M.G., Batistatou, A.K., Charalabopoulos, K.A., and Beris, A.E. 2007. The role of neurotrophins in axonal growth, guidance, and regeneration. Curr. Neurovasc. Res. 4:143-151.

34. Harada, C., et al. 2005. Role of neurotrophin- $4 / 5$ in neural cell death during retinal development and ischemic retinal injury in vivo. Invest. Ophthalmol. Vis. Sci. 46:669-673.

35. Calabrese, V., et al. 2005. Acetylcarnitine induces heme oxygenase in rat astrocytes and protects against oxidative stress: involvement of the transcription factor Nrf2. J. Neurosci. Res. 79:509-521.

36. Liu, J., Head, E., Kuratsune, H., Cotman, C.W. and Ames, B.N. 2004. Comparison of the effects of L-carnitine and acetyl-L-carnitine on carnitine levels, ambulatory activity, and oxidative stress biomarkers in the brain of old rats. Ann. N. Y. Acad. Sci. 1033: $117-131$

37. Calabrese, V., Giuffrida Stella, A.M., Calvani, M., and Butterfield, D.A. 2006. Acetylcarnitine and cellular stress response: roles in nutritional redox homeostasis and regulation of longevity genes. J. Nutr. Biochem. 17:73-88.

38. Virmani, A., Gaetani, F., and Binienda, Z. 2005. Effects of metabolic modifiers such as carnitines, coenzyme Q10, and PUFAs against different forms of neurotoxic insults: metabolic inhibitors, MPTP, and methamphetamine. Ann. N. Y. Acad. Sci. 1053:183-191.

39. Uchida, K., et al. 1998. Protein-bound acrolein: potential markers for oxidative stress. Proc. Natl. Acad. Sci. U. S. A. 95:4882-4887.

40. Shen, J.K., et al. 2007. Oxidative damage in agerelated macular degeneration. Histol. Histopathol. 22:1301-1308.

41. Gass, J.D., and Blodi, B.A. 1993. Idiopathic juxtafoveolar retinal telangiectasis. Update of classification and follow-up study. Ophthalmology. 100:1536-1546.

42. Ferrara, N. 2005. The role of VEGF in the regulation of physiological and pathological angiogenesis. EXS. 209-231.

43. Ferrara, N., and Kerbel, R.S. 2005. Angiogenesis as a therapeutic target. Nature. 438:967-974.

44. Adamis, A.P., and Shima, D.T. 2005. The role of vascular endothelial growth factor in ocular health and disease. Retina. 25:111-118.

45. Meyerle, C.B., et al. 2007. Intravitreal bevacizumab (Avastin) for retinal angiomatous proliferation. Retina. 27:451-457.

46. Charbel Issa, P., Holz, F.G., and Scholl, H.P. 2007. Findings in fluorescein angiography and optical coherence tomography after intravitreal bevacizumab in type 2 idiopathic macular telangiectasia. Ophthalmology. 114:1736-1742.

47. Bakri, S.J., and Ekdawi, N.S. 2008. Intravitreal triamcinolone and bevacizumab combination therapy for refractory choroidal neovascularization with retinal angiomatous proliferation. Eye. 22:978-980.

48. Kulkarni, A.C., Kuppusamy, P., and Parinandi, N. 2007. Oxygen, the lead actor in the pathophysiologic drama: enactment of the trinity of normoxia, hypoxia, and hyperoxia in disease and therapy. Antioxid. Redox Signal. 9:1717-1730.

49. Age-Related Eye Disease Study Research Group. 2001. A randomized, placebo-controlled, clinical trial of high-dose supplementation with vitamins $\mathrm{C}$ and $\mathrm{E}$ and beta carotene for age-related cataract and vision loss: AREDS report no. 9. Arch. Ophthalmol. 119:1439-1452.

50. Wang, J.Y., Wen, L.L., Huang, Y.N., Chen, Y.T., and $\mathrm{Ku}$, M.C. 2006. Dual effects of antioxidants in neurodegeneration: direct neuroprotection against oxidative stress and indirect protection via suppression of glia-mediated inflammation. Curr. Pharm. Des. 12:3521-3533.

51. Trommsdorff, M., et al. 1999. Reeler/Disabled-like disruption of neuronal migration in knockout mice lacking the VLDL receptor and ApoE receptor 2. Cell. 97:689-701.

52. Nusinowitz, S., and Heckenlively, J. 2006. Evaluating retinal function in the mouse retina with the electroretinogram. In Principles and practice of clinical electrophysiology of vision. J. Heckenlively, and G.B. Arden, editors. MIT Press. Cambridge, Massachusetts, USA. 189-206.

53. Gasmi, M., et al. 2007. Striatal delivery of neurturin by CERE-120, an AAV2 vector for the treatment of dopaminergic neuron degeneration in Parkinson's disease. Mol. Ther. 15:62-68

54. Besnard, F., et al. 1991. Multiple interacting sites regulate astrocyte-specific transcription of the human gene for glial fibrillary acidic protein. J. Biol. Chem. 266:18877-18883. 